ARTICLE

httpst//doi.org/10.1038/s41467-020-15809-5

\title{
Heat stress-induced transposon activation correlates with 3D chromatin organization rearrangement in Arabidopsis
}

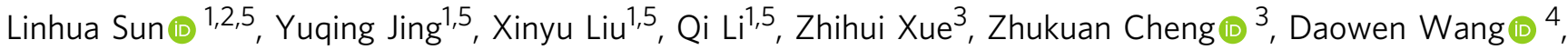 \\ Hang $\mathrm{He}{ }^{1 \times} \&$ Weiqiang Qian (10 ${ }^{1 凶}$
}

In higher eukaryotes, heterochromatin is mainly composed of transposable elements (TEs) silenced by epigenetic mechanisms. But, the silencing of certain heterochromatin-associated TEs is disrupted by heat stress. By comparing genome-wide high-resolution chromatin packing patterns under normal or heat conditions obtained through $\mathrm{Hi}-\mathrm{C}$ analysis, we show here that heat stress causes global rearrangement of the 3D genome in Arabidopsis thaliana. Contacts between pericentromeric regions and distal chromosome arms, as well as proximal intra-chromosomal interactions along the chromosomes, are enhanced. However, interactions within pericentromeres and those between distal intra-chromosomal regions are decreased. Many inter-chromosomal interactions, including those within the KNOT, are also reduced. Furthermore, heat activation of TEs exhibits a high correlation with the reduction of chromosomal interactions involving pericentromeres, the KNOT, the knob, and the upstream and downstream flanking regions of the activated TEs. Together, our results provide insights into the relationship between TE activation and 3D genome reorganization.

\footnotetext{
${ }^{1}$ State Key Laboratory of Protein and Plant Gene Research, School of Life Sciences, Peking University, Beijing 100871, China. ${ }^{2}$ Academy for Advanced Interdisciplinary Studies and Peking-Tsinghua Center for Life Sciences, Peking University, Beijing 100871, China. ${ }^{3}$ State Key Laboratory of Plant Genomics and Center for Plant Gene Research, Institute of Genetics and Developmental Biology, Chinese Academy of Sciences, Beijing 100101, China. ${ }^{4}$ College of Agronomy, State Key Laboratory of Wheat and Maize Crop Science, Henan Agricultural University, Longzi Lake Campus, Zhengzhou 450046, China. ${ }^{5}$ These

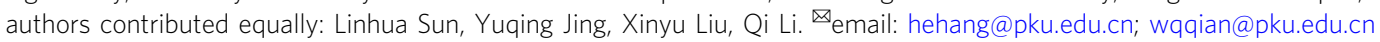


T he three-dimensional (3D) configuration of genome is dynamic and can regulate gene expression. Recently, Hi-C technology ${ }^{1}$, which couples proximity ligation-based chromosome conformation capture $(3 \mathrm{C})^{2}$, and next generation sequencing has revealed multifaceted and hierarchical 3D genome organization in microbes, animals, and plants (reviewed in ref. ${ }^{3}$ ). The Arabidopsis chromosomes, like mammalian chromosomes ${ }^{1}$, are organized into A and B compartments ${ }^{3,4}$. Notably, genome annotation shows that these two chromatin compartments are largely in accordance with the euchromatin/heterochromatin landscapes in Arabidopsis ${ }^{4}$.

Both Hi-C and fluorescence in situ hybridization (FISH) studies have identified a chromatin structure, the KNOT, in Arabidopsis ${ }^{4,5}$. The KNOT is formed by ten KNOT engaged elements (KEEs), or interactive heterochromatic islands, which are similar in interaction patterns to Piwi-interacting RNA clusters, the major controllers of transposable element (TE) silencing in Drosophila ${ }^{4-6}$. KEEs have either short- or long-range intrachromosomal and interchromosomal interactions ${ }^{4,5}$ and can also interact with telomeres ${ }^{4,5}$. Intriguingly, KEEs in euchromatic arms are enriched with the repressive histone mark H3K27me1 and small RNAs for gene silencing. TEs are preferentially integrated into KEEs, suggesting a role of the KNOT in TE defense ${ }^{4}$. A recent study indicates that KNOT can interact with invasive DNA elements, such as transgenes, and the interaction frequency is positively correlated with transgene silencing; this noncanonical silencing mechanism has been designated as KNOTlinked silencing 7 .

The knob, first discovered in maize ${ }^{8}$, is another conspicuous chromatin structure in Arabidopsis ${ }^{9}$. The knob hk4s in Arabidopsis, which is cytogenetically discernable, is about hundreds of kilobases close to the pericentromere on the left (top/short) arm of chromosome 4,10 . It consists of a low density of expressed genes but a high density of repeats derived from pericentromeric regions ${ }^{10} .4 \mathrm{C}$ (circular chromosome conformation capture) and Hi-C studies reveal that the knob contains high-frequency intraknob interactions that interact strongly with pericentromeric regions rather than with nearby euchromatic regions ${ }^{5,11}$. The fact that euchromatic regions do not participate in those strong interactions indicates that there is a mid-range chromosome loop connecting the knob to pericentromeric regions ${ }^{5,11}$.

A large fraction of the eukaryotic genomes is made up of TEs, which can cause mutations and genetic variations by changing their locations within a genome. TEs are usually silenced by DNA methylation, repressive histone modifications, and heterochromatin formation. Under prolonged heat stress, likely because heterochromatin-embedded genes important for heat response and heat tolerance need to be activated, a panel of TEs are transcriptionally activated ${ }^{12-14}$. Unlike TE activation under other conditions, TE activation under heat stress is not related to changes in DNA methylation, histone methylation, and histone acetylation. Changes in MOM1, a chromatin remodeler, and MORC6, a protein involved in transcriptional gene silencing and chromatin condensation, are also unnecessary for heat-induced TE activation ${ }^{12-15}$. However, Arabidopsis HIT4, a plant-specific regulator that translocates from the chromocenter to the nucleolus under heat stress ${ }^{16}$, is required for heat-induced TE activation ${ }^{17}$. A mediator subunit, MED14, and another transcription factor IIH subunit, UVH6, are also required for transcriptional activation of TEs under heat stress ${ }^{15}$. For the ONSEN family TEs, the presence of heat-responsive elements and binding of heat-responsive elements by heat shock factor A 2 are required for their activation ${ }^{18}$. After heat stress, the activated TEs are silenced and the CAF- 1 complex contributes to the resilencing of TEs during the recovery process ${ }^{13}$.
Here we explore the activation of Arabidopsis TEs in response to heat and resilencing of TEs during the recovery phase on a genome-wide scale. We unveil a strong correlation between chromatin decondensation and TE activation by heat through precisely mapping the changes of $3 \mathrm{D}$ chromatin organization using Hi-C analysis.

\section{Results}

Heat induces TE activation. To genome-wide identify heatactivated TEs, we sequenced the wild-type Col-0 transcriptome under Control, Heat, and Recovery conditions (Supplementary Fig. 1a and "Methods"). Principal component analysis (PCA) using the top 1,000 differentially expressed genes (DEGs) revealed good reproducibility between two biological replicates (Supplementary Fig. 1b). In total, 2,711 genes and 2,056 genes were significantly up- and downregulated, respectively, under Heat (Fig. 1a, Supplementary Fig. 1c, and Supplementary Data 1). Gene ontology (GO) analysis revealed that the upregulated genes showed enrichment for GO terms related to heat. The downregulated genes showed enrichment for GO terms related to temperature stimulus (Supplementary Fig. 1d and Supplementary Data 2). Specifically, many HEAT SHOCK PROTEIN (HSP) genes (e.g., HSP70 and HSP101) and small HEAT SHOCK PROTEIN genes (e.g., HSP21 and HSP18.2) were included in the significantly induced genes (Supplementary Fig. 1c). These results suggest the effectiveness of our heat treatment. Two well-studied epigenetically controlled protein coding genes (PCGs) SUPPRESSOR OF DRM1 DRM2 CMT3 (SDC) $)^{19}$ and QUA-QUINE $S T A R C H^{20}$ were induced under Heat (Supplementary Fig. 1c, e), suggesting that the induction of genes by heat stress could be related to epigenetic changes.

It is worth noting that more sequencing reads were assigned to TEs in Heat than that in Control (Supplementary Fig. 2a). Using the same criteria as we used to identify DEGs, we identified 363 upregulated but only 32 downregulated TEs (Fig. 1a and Supplementary Data 3). Thus, unlike the PCGs, which were almost equally upregulated and downregulated, TEs were predominantly activated by Heat (Fig. 1a). Most of the heatactivated TEs (268/363), representing the well-studied ONSEN ${ }^{21}$ and ROMANIAT5 22,23 TE families, were completely silenced in Control but showed dramatically high expression in heat (Fig. $1 \mathrm{~b}$ and Supplementary Fig. 2b). Retrotransposons, such as those in the Copia and Gypsy superfamilies of long terminal repeat (LTR) retrotransposons and short interspersed element non-LTR retrotransposons, were significantly overrepresented in heat-activated TEs (Fig. 1c, d). Conversely, DNA/MuDR and RC/Helitron transposon superfamilies were underrepresented in heat-activated TEs (Fig. 1c, d). Further TE family enrichment analysis revealed that, in total, 87 of $320 \mathrm{TE}$ families were overrepresented in heatactivated TEs (enrichment score $>1$ if absolute number $\geq 3$, see Supplementary Fig. 2c). ATCOPIA78, which is also known as $O_{O N S E N^{21}}$ and includes eight full-length ONSENs and a partial ONSEN, is the most enriched TE family (Supplementary Fig. 2c). Compared with all TEs, heat-activated TEs displayed genetic and epigenetic features including longer sizes, higher GC contents, and higher levels of heterochromatic histone mark H3K9me2 (Fig. 1e), suggesting that heat may preferentially activate heterochromatic transposons. Recently, it was reported that histone $\mathrm{H} 1$, the linker histone that binds to the nucleosome entry and exit sites of linker DNA, is required for heterochromatin condensation and perhaps involved in TE silencing ${ }^{24-26}$. Using published $\mathrm{H} 1$ chromatin affinity purification sequencing (ChAPSeq) and MNase-Seq datasets ${ }^{24,26}$ we found that, compared with random selected TEs, heat-activated TEs were enriched by H1.1 

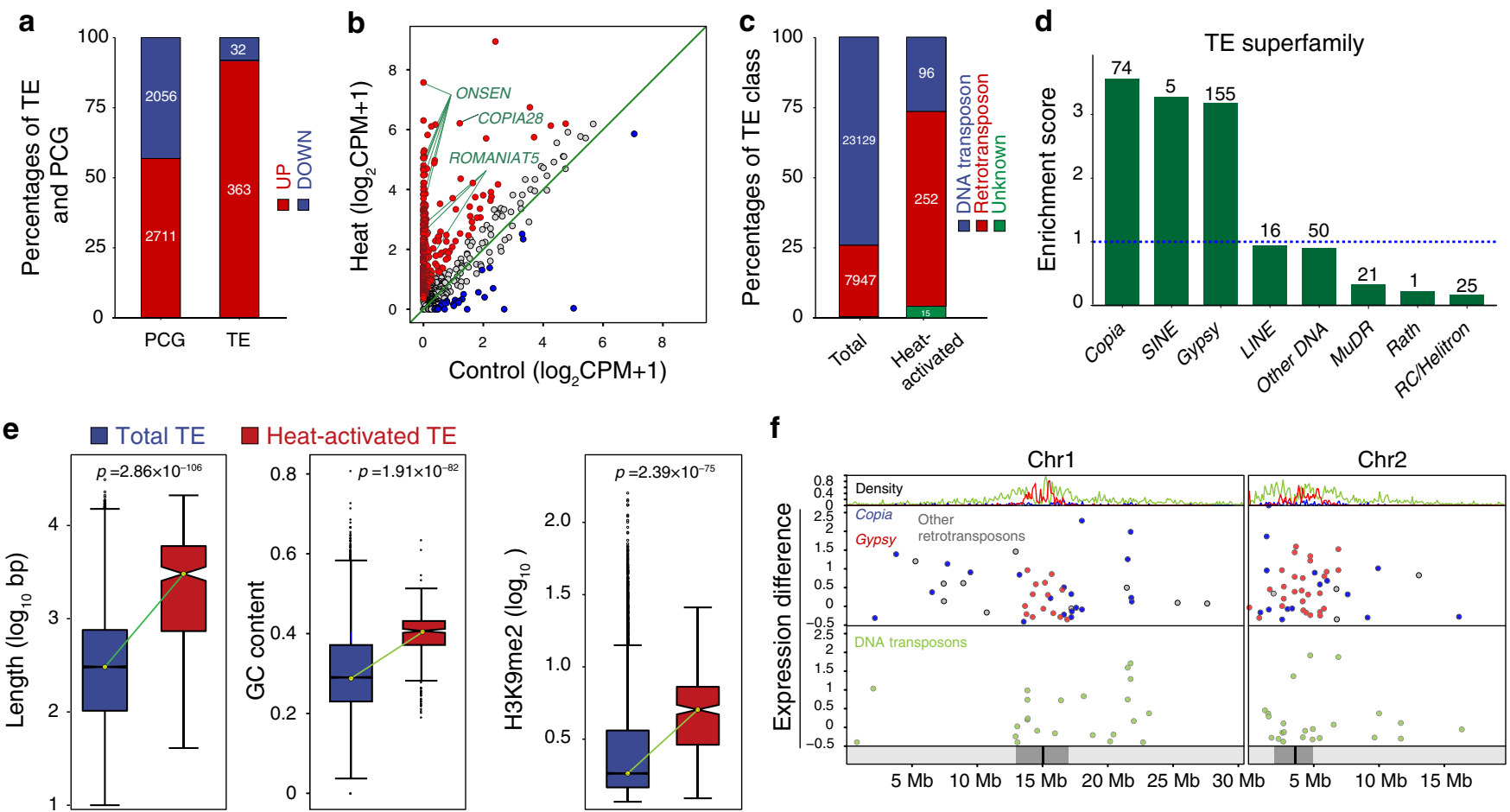

f

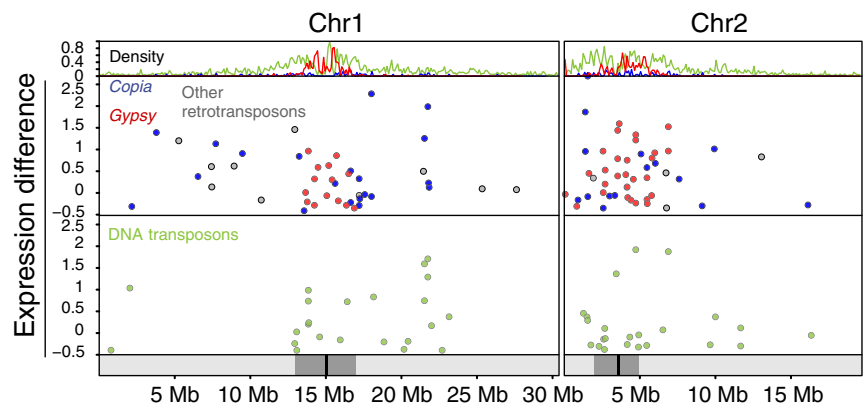

Fig. 1 Heat induces TE activation. a Percentages of up- and downregulated protein coding genes (PCGs) and transposable elements (TEs) under heat stress. Red bars: upregulation; Blue bars: downregulation. Numbers on the bars indicate the absolute number of TEs and PCGs. b Scatter plot of TE CPMs in heat vs. control conditions. CPM counts per million. Red dots: upregulated TEs under heat stress. Blue dots: downregulated TEs under heat stress. c The percentages of DNA transposons and retrotransposons for all (total) and for heat-activated TEs. Numbers marked on the bars indicate the absolute numbers of heat-activated TEs and total TEs from different TE classes. $\mathbf{d}$ The enrichment scores of TE superfamilies of heat-activated TEs. Each enrichment score was calculated as the ratio of the percentage of a certain TE superfamily in heat-activated TEs to that in all TEs. Numbers above the bars indicate the absolute numbers of heat-activated TEs in the corresponding superfamily. The blue dotted line marks the point of overrepresentation (enrichment score >1). e The lengths, GC contents, and H3K9me2 levels of TEs. The differences between heat-activated TEs and total TEs in the Arabidopsis genome was compared by a two-sided Mann-Whitney $U$ test for each feature. For each box plot, center lines indicate the medians; boxes show the 25th and 75th percentiles; whiskers extend to the minimum and maximum. $\mathbf{f}$ Chromosomal distributions of heat-activated TEs in chromosomes 1 and 2. The uppermost track shows the density of Copia (blue) and Gyspy (red) retrotransposon superfamilies and DNA transposons (green). The two tracks in the middle show the distribution of retrotransposons and DNA Transposons. Expression differences were calculated by $\log _{10}\left(\mathrm{Heat}_{\mathrm{CPM}}-\mathrm{Contro}_{\mathrm{CPM}}\right.$ ). TEs belonging to the Copia and Gyspy retrotransposon superfamilies and DNA transposons are colored in blue, red, and green, respectively. Other retrotransposons are colored in gray. The bottom track shows the positions of centromeres (black) and pericentromeres (gray).

and H1.2 and were also occupied by large numbers of nucleosomes (Supplementary Fig. 2d).

Furthermore, a large number (188) of heat-activated TEs were located in pericentromeric and centromeric regions (Fig. If and Supplementary Fig. 2e). According to a Fisher's exact test (among 21,125 TEs on chromosomes arms, 175 are heat-activated. Among 10,064 TEs in pericentromeric and centromeric regions, 188 are heat-activated. The $p$-value is $1.096 \mathrm{E}-14)$, the enrichment of heat-activated TEs in pericentromeric and centromeric regions is a true bias (pericentromeric and centromeric regions were defined previously ${ }^{4}$ ). The heat-activated Gypsy superfamily of retrotransposons (155/363) were particularly centered around centromeres. Also, heat-activated retrotransposons in the Copia superfamily (23/363) and DNA transposons (41/363) were concentrated in pericentromeric regions, although others were dispersed within euchromatin (Fig. If and Supplementary Fig. 2e). Our results were consistent with previous microarray results ${ }^{14}$.

Under Recovery, 319 out of 363 heat-activated TEs were resilenced, while the rest remained activated (Fig. 2a). The 319 TEs that were silenced again were referred to as Group 1 TEs and other heat-activated TEs were referred to as Group 2 TEs (Fig. 2a). We validated the expression patterns of representative Group 1 and Group 2 TEs by RT-qPCR (Fig. 2b and Supplementary Fig. 3a, b). We then compared the epigenetic features of Group 1 and Group 2 TEs using publicly available data (see "Methods") and found that the density of active histone marks, such as H4K16ac, H3K36ac, H3K56ac, H3K36me3, and H3K4 methylation, was higher, but the density of repressive histone marks, such as $\mathrm{H} 3 \mathrm{~K} 9 \mathrm{me} 2$ and $\mathrm{H} 3 \mathrm{~K} 27 \mathrm{me}$, was lower in Group 2 TEs. This may explain why the activation of Group 2 TEs persisted under Recovery (Fig. $2 c$ and Supplementary Fig. 3c).

Heat induces rearrangement of chromatin organization. Previous FISH studies revealed that heterochromatin decondensation accompanies the activation of TEs under prolonged heat stress ${ }^{13}$ In our study, H3K27me1 immunostaining results further confirmed that heterochromatin underwent decondensation under Heat and recondensed under Recovery (Supplementary Fig. 4a,b). Furthermore, the nuclei became bigger under Heat (Supplementary Fig. 4c). To test whether chromatin organization rearrangement and TE release under Heat are correlated, we precisely mapped the changes of 3D chromatin organization in response to heat by performing Hi-C experiments using Arabidopsis under Control and Heat conditions (Supplementary Data 4 and "Methods"). In total, we detected more than 170 and 195 million interaction pairs under Control and Heat, respectively. The ratios 


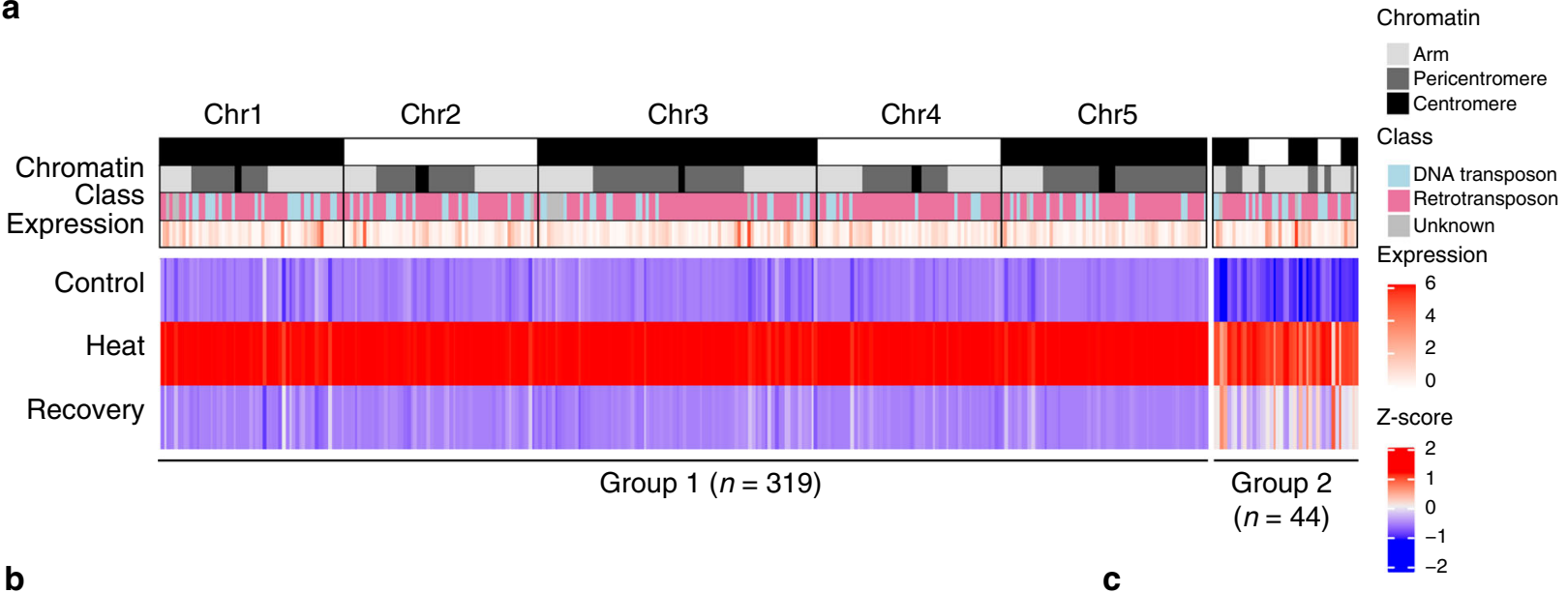

b

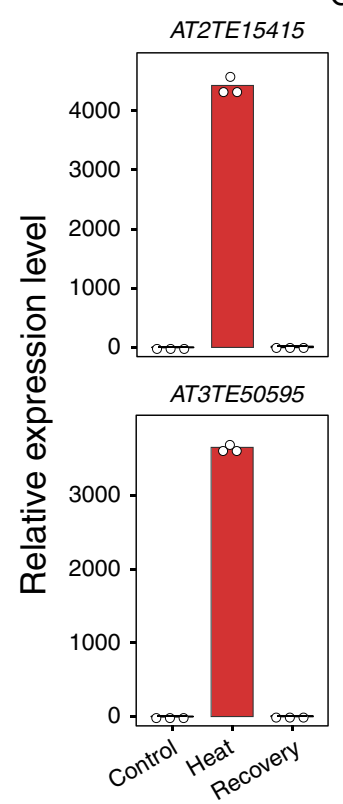

Group 1
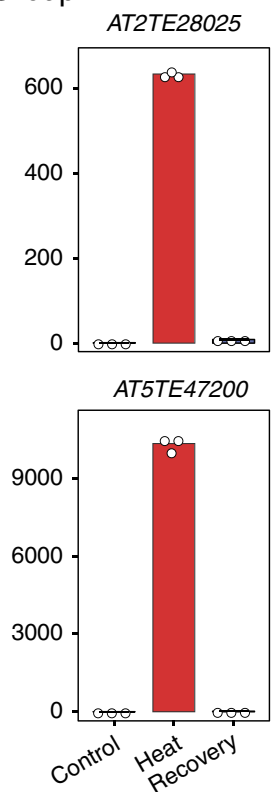

Group 2

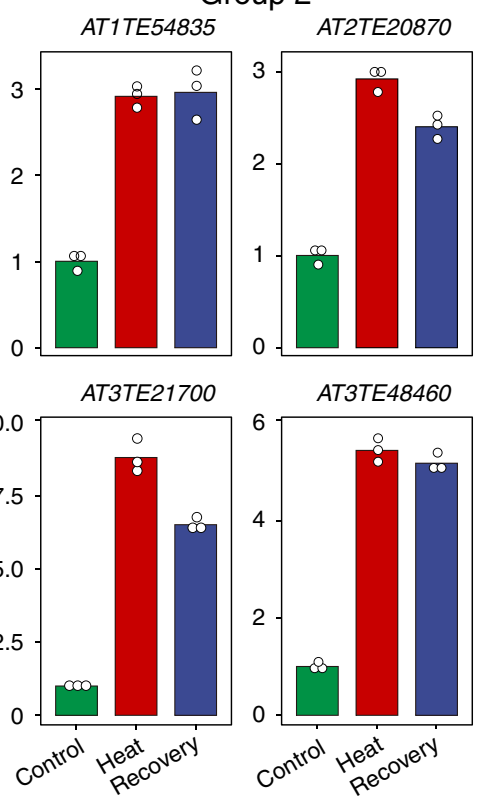

C

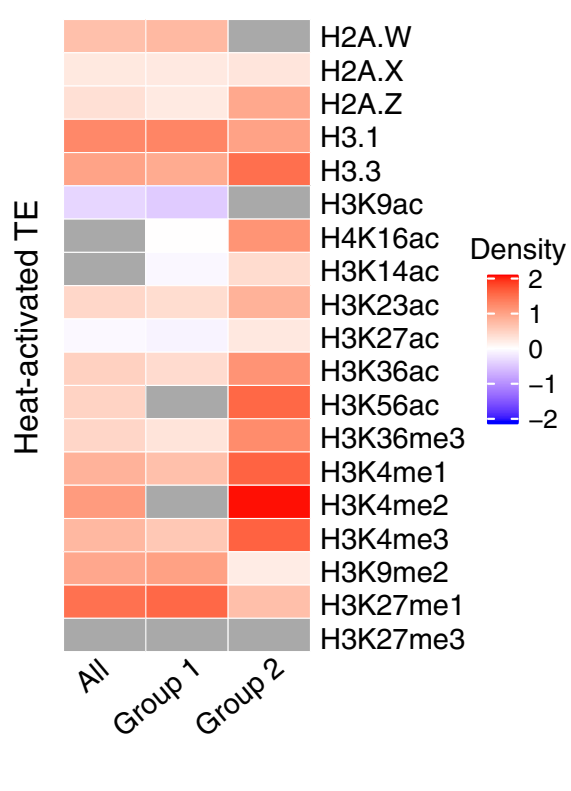

Fig. 2 Characterization of two groups of heat-activated TEs. a Heatmap of heat-activated TEs in Control, Heat, and Recovery. The chromatin track shows the genomic locations of heat-activated TEs. The class track shows the three classes of the heat-activated TEs. The expression track shows the expression levels of heat-activated TEs in $\log _{2}(C P M+1)$. The bottom three tracks show relative expression levels (Z-scores) for Control, Heat, and Recovery and range from -2 (blue) to 2 (red). Most heat-activated TEs, classified into Group 1, are silenced again during recovery. Some heat-activated TEs, classified into Group 2, exhibited persistent activation or were not completely restored during recovery. $\mathbf{b}$ Expression dynamics of randomly selected Group 1 and Group 2 TEs as determined by RT-qPCR. The expression value of each replicate was normalized to the expression level of Col-0. Circles denote relative expression values. Bars indicate mean values of three technical replicates. c The enrichment (red) and depletion (blue) patterns of epigenetic features in Group 1 and Group 2 TEs compared with total TEs in Arabidopsis. Relative density ranges from -2 to 2 . Gray indicates that the epigenetic feature is neither enriched nor depleted (two-sided Mann-Whitney $U$ test, $p>0.05$ ). Source data underlying $\mathbf{b}$ and $\mathbf{c}$ are provided as a Source Data file.

of cis (intrachromosomal) to total valid interactions, which are positively correlated with $\mathrm{Hi}-\mathrm{C}$ quality ${ }^{27}$, reaches $83.4 \%$ (Control) and $87.5 \%$ (Heat) in our Hi-C datasets, similar to the ratios from in situ $\mathrm{Hi}-\mathrm{C}^{28}$ and much higher than those from dilution Hi$\mathrm{C}^{4,5,29-31}$ of Arabidopsis, thus indicating the high quality of our $\mathrm{Hi}$-C. Genome-wide and chromosome-wide comparisons of eigenvalues, correlation analysis of eigenvalues of the first principal component (PC1) (Supplementary Fig. 5a,b) and direct comparisons of the Hi-C interaction heatmaps of two biological replicates (Supplementary Fig. 5c,d) revealed good reproducibility of our Hi-C. To further assess the reliability of our $\mathrm{Hi}-\mathrm{C}$, we compared our Control Hi-C datasets with five previously published Hi-C datasets of wild-type Arabidopsis thaliana ${ }^{4,5,29-31}$. Correlation and enrichment analyses of eigenvalues of the PC1 to various genomic and epigenomic features validated the similarity $(r=0.99)$ between our Control dataset and other Hi-C datasets (Supplementary Fig. 6). Finally, by using the pipeline of landmark $\mathrm{Hi}-\mathrm{C}$ research ${ }^{32}$, the resolution of our $\mathrm{Hi}-\mathrm{C}$ data was estimated to be 0.95 and $0.85 \mathrm{~kb}$ for Control and Heat, respectively (Supplementary Data 4 and "Methods"), which were only reached by a Hi-C map constructed by merging several datasets of Arabidopsis ${ }^{31}$.

We then explored genome-wide chromatin interaction patterns of Control and Heat at $100 \mathrm{~kb}$ resolution (see "Methods"). Like Hi-C maps constructed for other species and cell types ${ }^{3}$, our Hi-C maps for Arabidopsis under Control and Heat conditions show frequent chromatin interactions along the diagonal (Supplementary Fig. 7). These chromatin interaction patterns can be explained by the existence of chromosome territories ${ }^{27}$. Prominent interactions were 
a

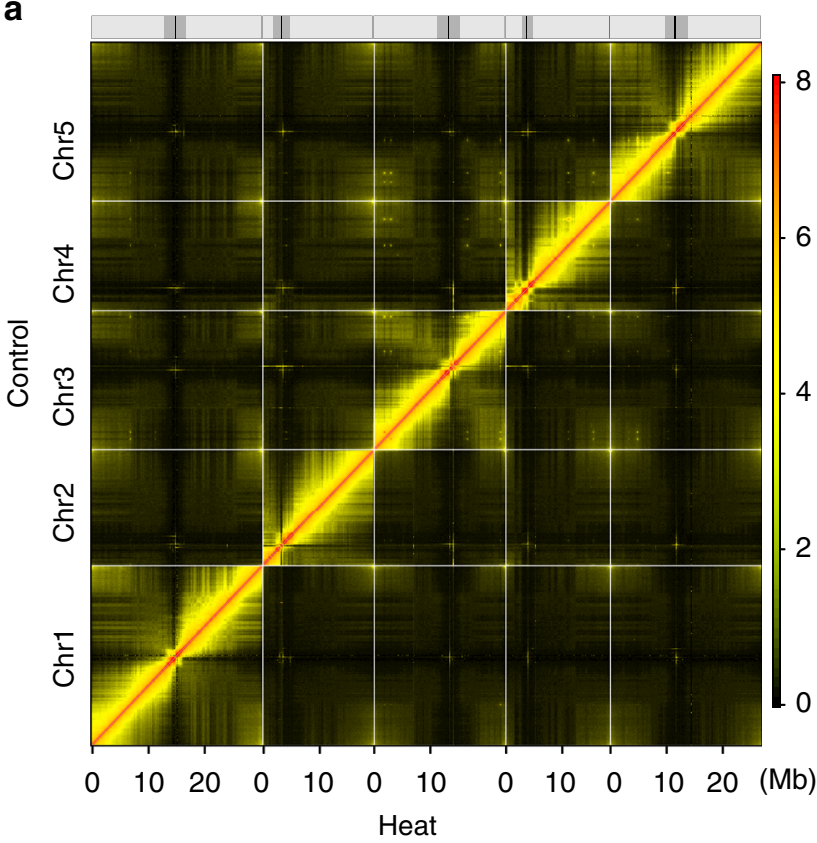

b

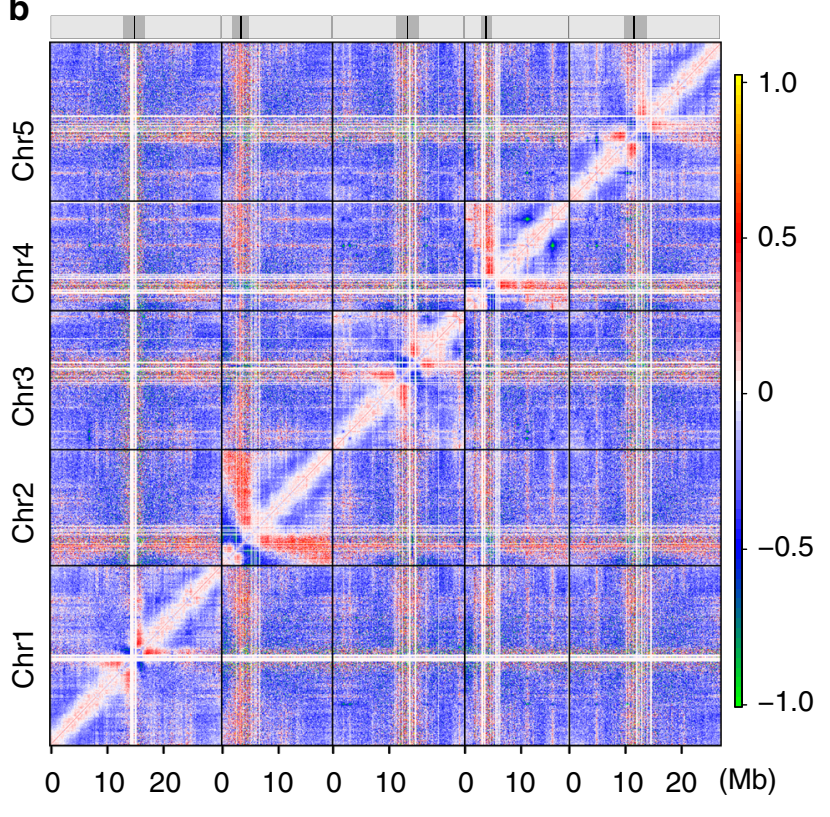

C

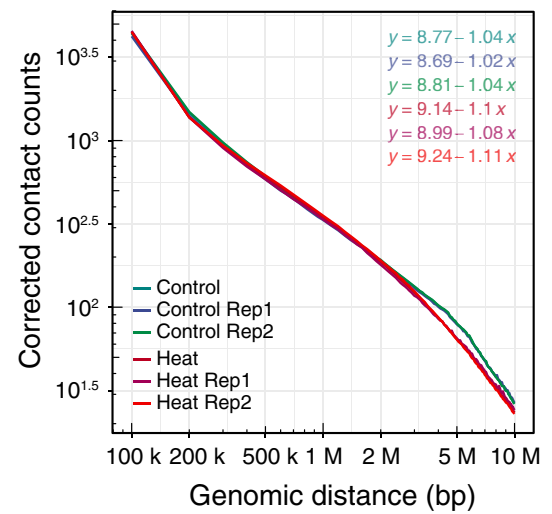

d

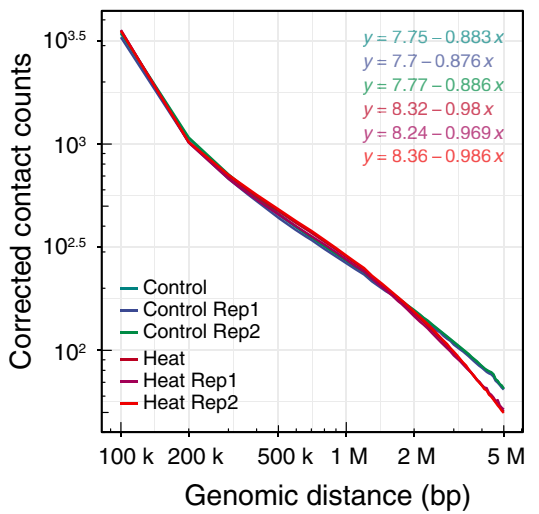

e

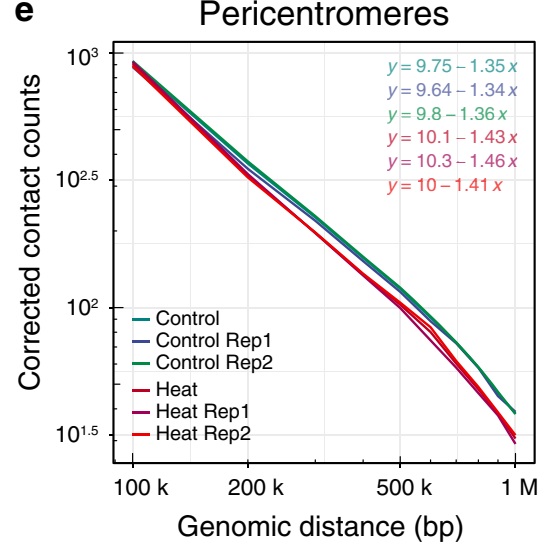

Fig. 3 Heat induces rearrangement of chromatin organization. a Genome-wide chromatin organization revealed by a Hi-C interaction frequency heatmap at $100 \mathrm{~kb}$ resolution for all Arabidopsis chromosomes in Control and Heat. Each pixel denotes all interactions between any two $100 \mathrm{~kb}$ genomic loci from the linear genome. Intensity represents $\log _{2}$ normalized contact frequencies. The top track shows the positions of centromeres (black) and pericentromeres (gray). b Genome-wide heatmap of relative interaction differences between Heat and Control. The relative difference is calculated as the difference of two interaction frequencies divided by the mean of two interaction frequencies. In the plot, Hi-C interactions that become stronger (chromosome condensation) in Heat compared with Control are in red to orange, while Hi-C interactions that become weaker (chromosome loosening) in Heat compared with Control are in blue to green. Hi-C interactions that do not change in Heat compared with Control are in white. The genomic bin size is 100 $\mathrm{kb}$ and the upper- and lower-triangular matrices are symmetric. The top track shows the positions of centromeres (black) and pericentromeres (gray). c Averaged scaling plot of interaction frequencies against increasing genomic distance for all Arabidopsis chromosomes. The genomic bin size is 100 kb. $\mathbf{d}$ Averaged scaling plot of interaction frequencies against increasing genomic distance for all Arabidopsis chromosome arms. The genomic bin size is 100 kb. e Averaged scaling plot of interaction frequencies against increasing genomic distance for all Arabidopsis pericentromeric regions. The genomic bin size is $100 \mathrm{~kb}$.

observed within the pericentromeric regions, among different pericentromeric regions, and among telomeres (Supplementary Fig. 7), which were consistent with previously published Hi-C findings ${ }^{5}$.

To investigate the effects of heat stress on global chromatin organization, we calculated relative interaction differences at 100 $\mathrm{kb}$ resolution (Fig. 3a, b and Supplementary Fig. 8), where a relative interaction difference was defined as the difference of two interactions divided by the mean of the two interactions ${ }^{4,29,33}$. Visual inspection of the map of relative interaction differences revealed prominently enhanced interactions between pericentromeric regions and distal regions of the chromosome arms in the genome, especially in chromosomes 2 and 4 under Heat, while slightly altered proximal intrachromosomal interactions were found along the chromosomes (Fig. $3 \mathrm{~b}$ and Supplementary Fig. 8a-e). However, interactions within pericentromeres were greatly reduced. Both distal intrachromosomal interactions and many interactions between different chromosome arms were also reduced (Fig. 3b and Supplementary Fig. 8a-e). These changes can be observed in both biological replicates (Supplementary Fig. 8f). Thus, heat stress could cause global genome organization rearrangement, altering proximal interactions within chromosome arms, as well as interactions of chromosome arms with pericentromeric regions. 
Previous research suggested that the average interaction frequency between any two loci decays in a power-law function as the genomic distance increases ${ }^{4}$. To examine this, we generated scaling plots of interaction frequencies against genomic distance for different regions in Control and Heat at $100 \mathrm{~kb}$ resolution. Scaling plot results indicated that heat stress caused a decrease of long-range chromatin interactions, which are far away from the diagonal in the Hi-C heatmap (Fig. $3 \mathrm{c}-\mathrm{e}$ and Supplementary Fig. 9a-c). However, the short-range chromatin interactions, which are near the diagonal, were slightly enhanced at $1 \mathrm{~kb}$ resolutions (Supplementary Fig. 10).

To quantitatively assess heat-induced chromatin organization changes, we calculated interaction decay exponents (IDEs), which were defined as the slopes of a linear fit of mean interaction intensities detected at a given range ${ }^{1}$, that represent the distancedependent decay of interaction frequency at chromosome arms, chromosomes, and pericentromeres (Fig. 3c-e and Supplementary Fig. 9d). IDEs of chromosomes, chromosome arms, and pericentromeres in Control were $-1.04,-0.88$, and -1.35 , respectively, and comparable with previously calculated IDEs in Arabidopsis ${ }^{4}$ (Supplementary Fig. 9d). However, IDEs of chromosomes, chromosome arms, and pericentromeres in Heat were -1.10 , -0.98 , and -1.43 , respectively (Supplementary Fig. 9d). In general, heat-stressed chromatin exhibited smaller IDEs in different genomic compartments (Supplementary Fig. 9d).

Chromatin compartmentation is weakened under heat stress. Active (A) and inactive (B) compartments in Arabidopsis can be identified by bidirectional PC1 values ${ }^{4}$. Comparison of PC1 values in Control and Heat (Supplementary Fig. 11) revealed that heat only induces minimal $\mathrm{A}$ and $\mathrm{B}$ compartments switching (Fig. 4a and Supplementary Fig. 11). However, chromatin compartmentation was weakened by heat stress (Fig. 4b, c). The weakening of chromatin compartmentation was prominent in pericentromeric regions and the knob ( $h k 4 s$ ) (Fig. 4d and Supplementary Fig. 11). The weakening of chromatin compartmentation was associated with an decrease in chromatin interactions within the same compartment (Fig. 4b).

Interactions between KEEs are reduced under heat stress. Intriguingly, the signals for KNOTs, which are formed by chromatin interactions among KEEs, were remarkably reduced (Fig. 4d). We used $\mathrm{HOMER}^{34}$ to calculate pairwise interaction scores of the ten KEEs in the Arabidopsis genome at $1 \mathrm{~kb}$ resolution. Most (29/45) interaction scores between KEEs were reduced by at least $50 \%$ (Fig. $5 \mathrm{a}$ ). The reduction was most prominent for interaction scores between KEE7 and KEE8 (Fig. 5a-c), which was highest in Control. As a pair, KEE3 and KEE4, which had the second-highest interaction frequency under Control, also underwent dramatic interaction score reductions under Heat (Fig. 5a, c). To validate changes of interaction frequencies between KEEs, we performed 3C experiments. Our results showed that the relative interaction frequency between KEE3 and KEE4 was significantly decreased in Heat compared with that in Control (Fig. 5d). Their interaction was partially recovered in Recovery (Fig. 5d), suggesting that the interaction frequency decrease between KEE3 and KEE4 was reversible. We also performed FISH experiments to analyze the association rates of KEE3/KEE4 and KEE7/KEE8 and found those rates to be significantly decreased under Heat and recovered under Recovery (Fig. 5e and Supplementary Fig. 12). Together, our results suggest that the KNOT is a dynamic structure.

Heat stress causes extensive knob related chromatin decondensation. Zooming in on the first 6 megabases of chromosome
4, we also found two distinctly reduced chromatin interactions in the heatmap. First, chromatin interactions within the knob (Chr4:1,560,561-2,082,885) in chromosome 4 showed a distinct decrease, which can be seen visually (Supplementary Fig. 13). Then, a virtual $4 \mathrm{C}$ profile generated from the $\mathrm{Hi}-\mathrm{C}$ contact matrix was plotted from a site (Chr4:1,760,000-1,780,000) within the knob that contains a previously reported viewpoint (Chr4:1,765,891) from 4C ${ }^{11}$ (Supplementary Fig. 13). A virtual $4 \mathrm{C}$ profile, as well as a heatmap, demonstrated that a mid-range chromosome loop, possibly mediated by long-range chromatin interactions between the knob and the right-half of the pericentromere (Chr4:3,856,518-5,000,000), had remarkably reduced interaction intensities in Heat (Supplementary Fig. 13). Our results indicated that, like the KNOT interactions, the interactions within the knob and interactions between the knob and the right-half of the pericentromere were also reduced under Heat.

Heat activation of TEs rises as chromatin interactions drop. To evaluate the relationship between the activation of TEs and chromatin organization changes in response to heat stress, we compared changes of TE expression levels with changes in chromatin compartmentalization. The activation of TEs often coincided with an increase in $\mathrm{PC} 1$ value in Heat condition relative to Control condition in heterochromatin (Fig. 4d and Supplementary Fig. 11). Specifically, among 156 heat-activated TEs in euchromatin, only $51(32.7 \%)$ were associated with an increase in PC1 value. However, among 209 heat-activated TEs in heterochromatin, 135 (64.6\%) were associated with an increase in PC1 value. For heat-activated TEs in pericentromeric regions and the knob, the percentage was even higher. We further compared changes of TE expression levels with changes in knob-mediated interactions. Heat-induced TE activation correlated with reduced chromatin interactions within the knob and the mid-range chromosome loop between the knob and the left pericentromere of chromosome 4 under heat stress. Eleven heat-activated TEs resided in regions that form the knob (Supplementary Fig. 13 and Supplementary Data 3).

Local chromatin interaction changes associate with TE activation. PCA analysis used $20 \mathrm{~kb}$ bin, which is much longer than lengths of TEs, including heat-activated TEs (Fig. 1e). Comparison between local chromatin organization changes and heat-induced TE activation is necessary to better determine the level of correlation between chromatin reorganization and heat-induced TE activation. To this end, we first analyzed the relationship between heat-activated TEs and small chromatin loops (2-25 kb scale) identified from a previous study ${ }^{31}$. These small chromatin loops were identified from Arabidopsis genome except regions near centromeres (Chr1:13,698,787-15,897,560; Chr2:2,450,0025,500,000; Chr3:11,298,762-14,289,014; Chr4:1,800,001-5,150,000; Chr5:10,999,995-13,332,770). We determined whether heatactivated TEs overlap with loops, that is whether a TE body overlaps with one end (anchor) of a chromatin loop or a TE body is inside a chromatin loop. We found that 131 heat-activated TEs overlapped with at least one chromatin loop (Supplementary Data 5). The results suggest the existence of local chromatin structures over most heat-activated TEs. Interestingly, when we order heat-activated TEs by the numbers of their overlapped chromatin loops, we found that many TEs overlapped with dozens of chromatin loops (Supplementary Data 5). For example, AT3TE13060 from KEE4 and AT5TE17345 from KEE9 overlapped with $\sim 30$ chromatin loops.

Secondly, to show fine-scale chromatin organization changes upon heat, we generated $\mathrm{Hi}-\mathrm{C}$ chromatin interaction map (in 
a

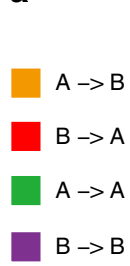

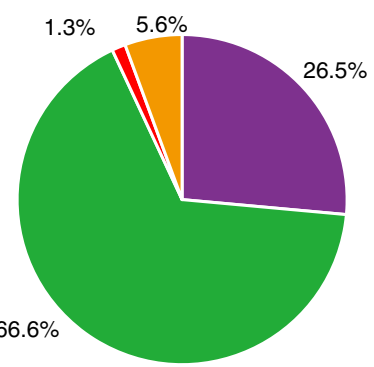

b

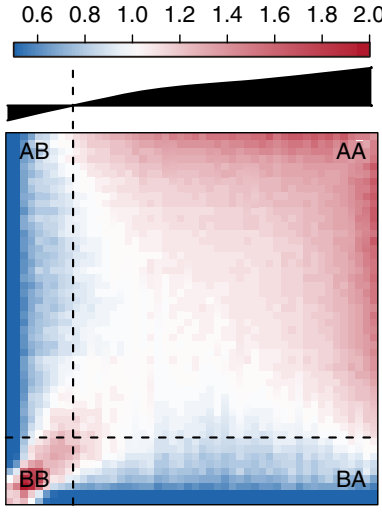

Control

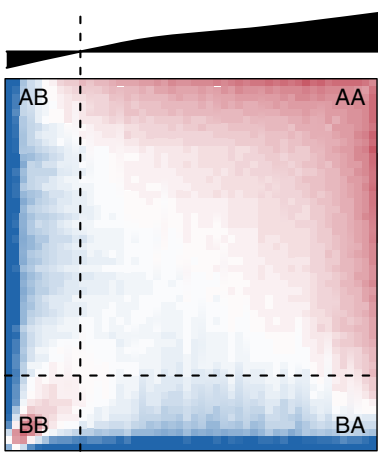

Heat d

\begin{tabular}{lllll}
0 & 2 & 4 & 6 & 8 \\
\hline
\end{tabular}

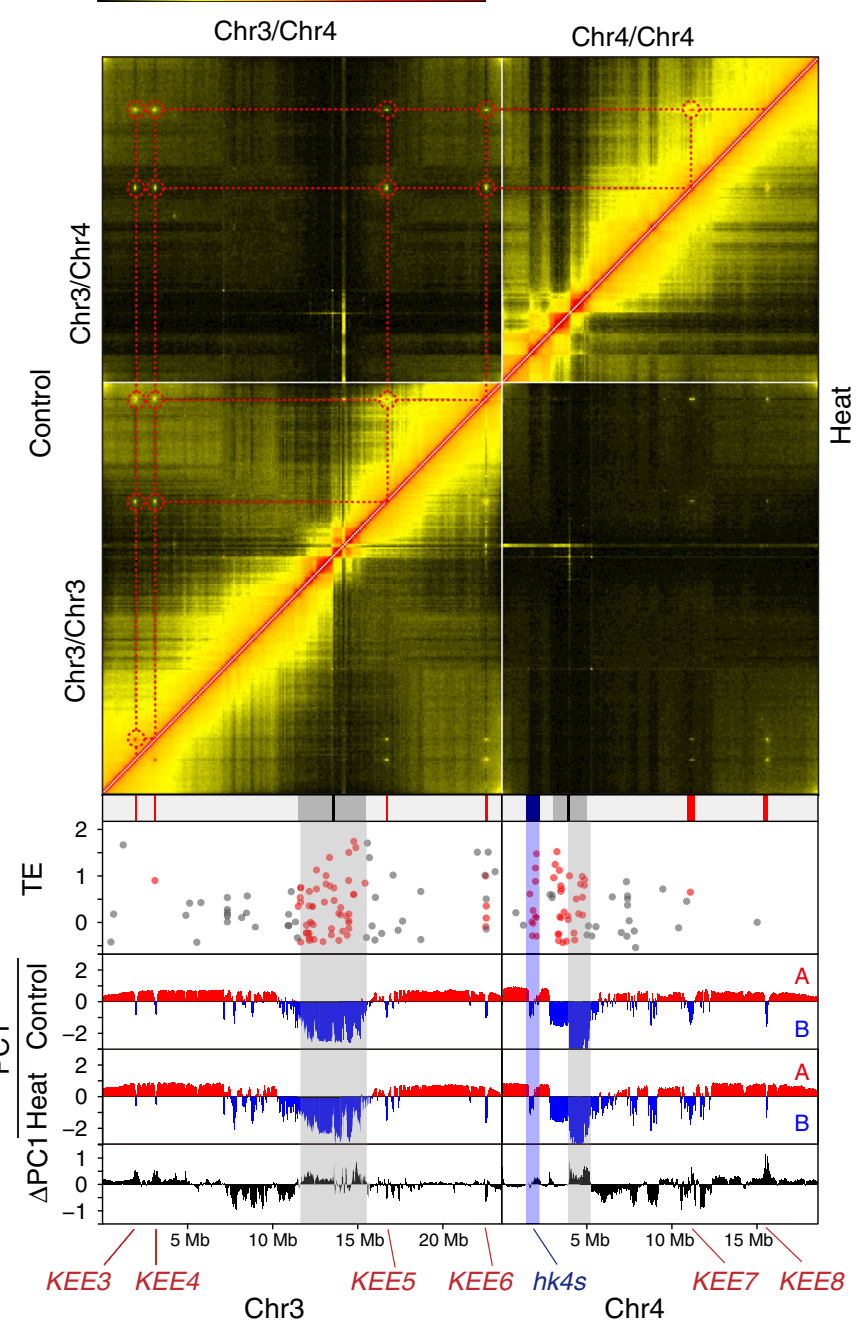

Heat-activated TE

- Located within heterochromatin

- Located within euchromatin

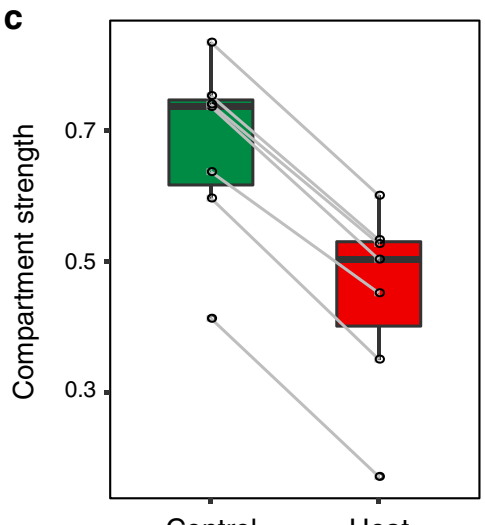

Control Heat

Fig. 4 Heat weakens chromatin compartmentation. a Pie chart showing the percentages of chromatin compartment switching induced by heat. A compartment: active compartment; B compartment: repressive compartment. b Saddle plots of chromatin compartmentalization: mean cis observed interaction frequencies divided by expected interaction frequency between $20 \mathrm{~kb}$ bins. The plots are ordered by PC1 values from Control. Interactions between A compartments are in the top right, and interactions between B compartments are in the bottom left. c Compartment strengths in Control and Heat for each chromosome arm split by centromere. Compartment strength is defined as natural logarithm of interactions of $A A+B B$ normalized by interactions of $A B$. For the box plot, center lines indicate the medians; boxes show the 25th and 75th percentiles; whiskers extend to the minimum and maximum. d Heatmap showing contact frequencies between and within chromosomes 3 and 4 in Control and Heat. The upper- and lower-triangular matrices correspond to Control and Heat, respectively. The values on the diagonal line (interactions between nearby bins) are assigned to zero. The KEEs are highlighted by dashed, red circles and interconnected by dashed lines to indicate the relationships with each other and the positions of KEEs in the linear genome. The track below shows the positions of centromeres (black), pericentromeres (gray), the knob hk4s (blue), and KEEs (red). The lower panel shows the positions and differential expression of heat-activated TEs within chromosomes 3 and 4, PC1 values in Control condition, PC1 values in Heat condition, and $\triangle P C 1$ values $\left(\mathrm{PC}_{\text {Heat }}-\mathrm{PC1}\right.$ Control $)$ horizontally. The positive $\mathrm{PC1}$ values indicate $\mathrm{A}$ compartments. The negative $\mathrm{PC1}$ values indicate $\mathrm{B}$ compartments. The positions of pericentromeres and the knob $h k 4 s$ with increased PC1 values are indicated. Source data underlying $\mathbf{a}$ and $\mathbf{d}$ are provided as a Source Data file.

Knight and Ruiz (KR) balanced matrix and observed/expect matrix which eliminates distance effects) at $1 \mathrm{~kb}$ resolution. We found prominent chromatin interaction changes at specific loci like KEE3, KEE4, KEE6, and KEE9 (Fig. 6a, b and Supplementary Fig. 14a-c). We examined TE expression changes at these loci. We found that TE activation was correlated with chromatin organization changes (Fig. 6a, b and Supplementary Fig. 14c). It should be noted that these loci contain heat-activated TEs overlapping with large numbers of chromatin loops (Fig. 6a, b and Supplementary Fig. 14).

Thirdly, we found that small triangles are widespread and prominent in Arabidopsis $\mathrm{Hi}-\mathrm{C}$ map at $\mathrm{kb}$ level resolution (Fig. 6a, b and Supplementary Fig. 14), indicating the prevalence of local 3D chromatin structures in Arabidopsis. These small triangles overlapped with previously identified chromatin loops ${ }^{31}$. Those triangles/chromatin loops were not topologically 
a

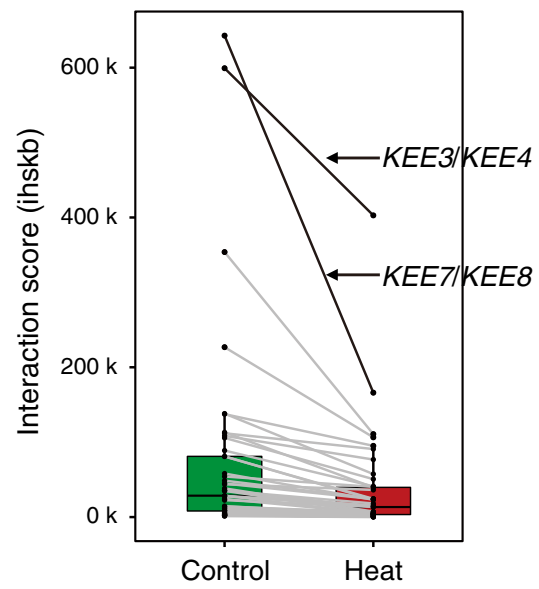

C

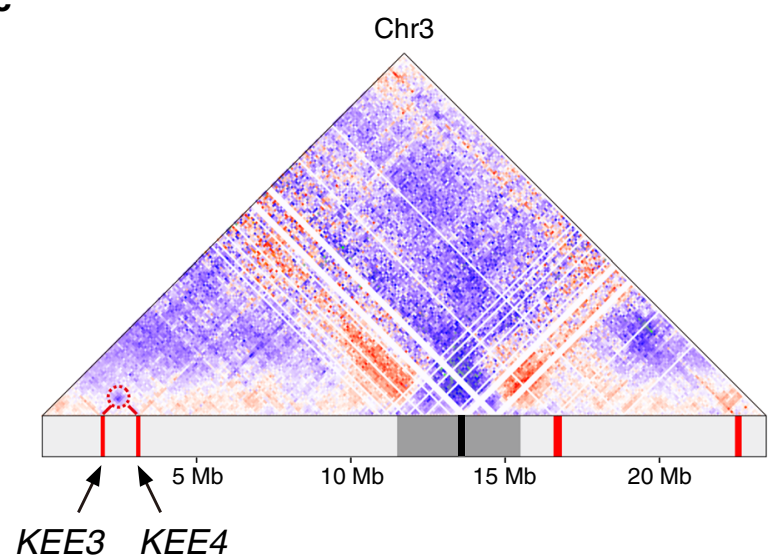

b

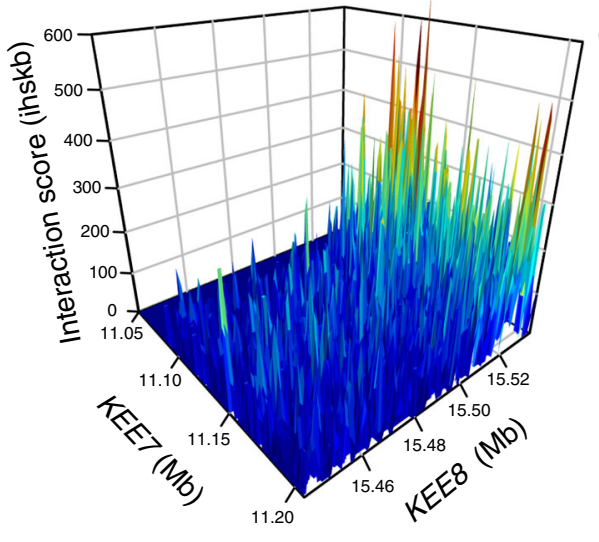

Control

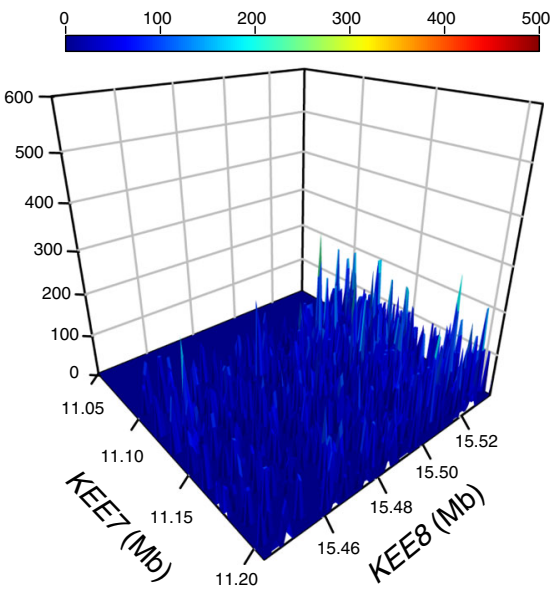

Heat

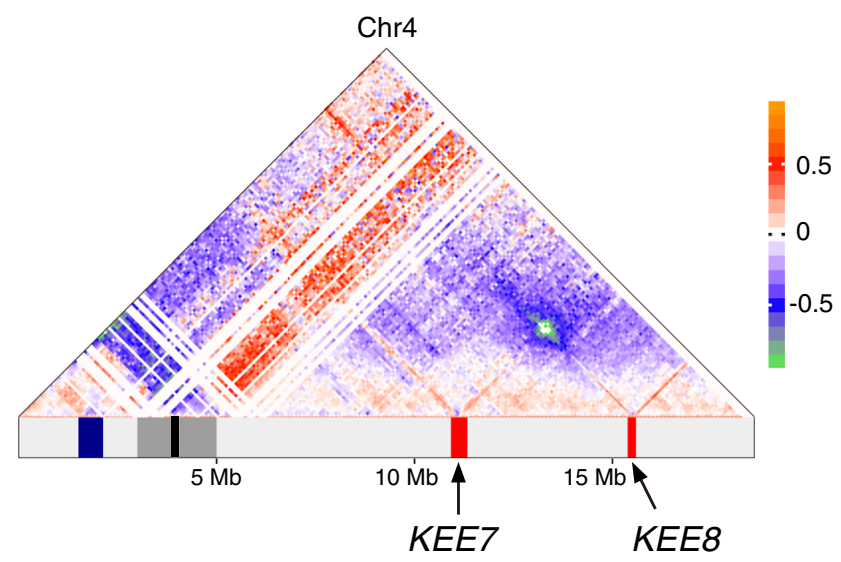

d
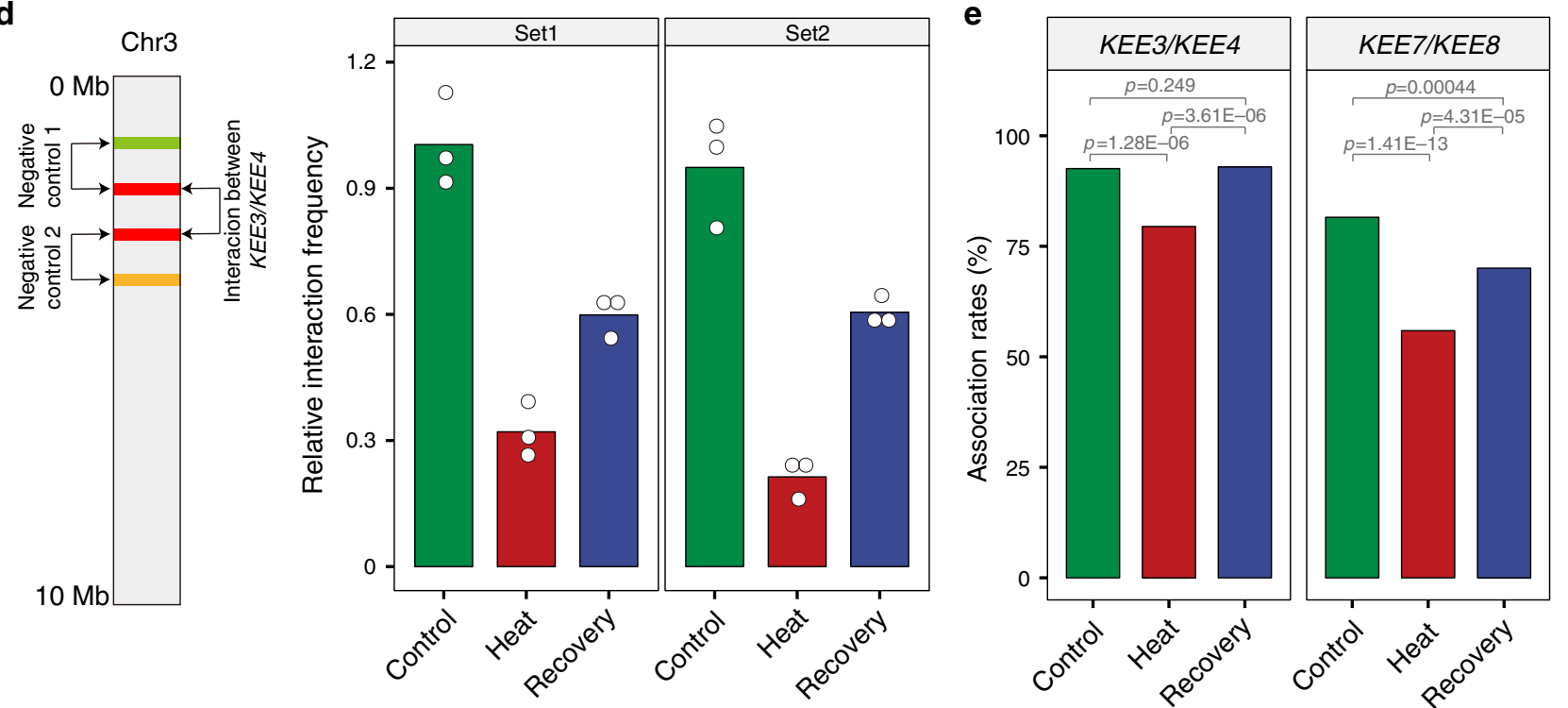

associating domains (TADs). They were more like "crumples" defined in yeast by Micro- $\mathrm{C}^{35}$. However, because they were similar to TADs in shape, we applied the TAD-separation score method to analyze Arabidopsis Hi-C map. The TAD-separation score method has been used to define TADs. Regions with local higher TAD-separation scores are considered to be TAD bodies $^{36}$. We could observe clear triangle structures associated with local peaks of TAD-separation score (Fig. 6a, b and Supplementary Fig. 14). A meta-TE analysis of TAD-separation scores over heat-activated TEs also suggested the existence of local triangle structures over heat-activated TEs (Fig. 6c, d).

Fourthly, to further study the relationships between $\mathrm{TE}$ expression and chromatin organization change at a genomewide scale, we analyzed the chromatin interaction changes 
Fig. 5 Reduced interactions among KEEs under heat stress. a Pairwise interaction scores of all ten KEEs in Control and Heat, defined as the interactions per hundred square kilobases per billion mapped reads (ihskb). KEE3/KEE4 and KEE7/KEE8 are highlighted. For each box plot, center lines indicate the medians; boxes show the 25th and 75th percentiles; whiskers extend to the minimum and maximum. b Perspective plots of the interactions between KEE7 and KEE8 in Control and Heat. The $X$-axis and Y-axis show the genomic positions of KEE7 (Chr4: 11,050,000-11,206,537) and KEE8 (Chr4: 15,441,465-15,537,500). The Z-axis shows the interaction scores. c Triangle heatmaps of the relative differences between Heat and Control in chromosomes 3 and 4 . $\mathrm{Hi}-\mathrm{C}$ interactions that become stronger (chromosome condensation) in Heat than those in Control are in red to orange, while $\mathrm{Hi}-\mathrm{C}$ interactions that become weaker (chromosome loosening) in Heat than those in Control are in blue to green. $\mathrm{Hi}-\mathrm{C}$ interactions that do not change in Heat compared with Control are in white. The genomic bin size is $100 \mathrm{~kb}$. The interactions of KEE3/KEE4 and KEE7/KEE8 are highlighted with circles. The bottom track shows the positions of centromeres (black), pericentromeres (gray), the knob hk4s (blue), and KEEs (red). d $3 C$ results showing the significantly decreased interactions between KEE3 and KEE4 in Heat compared with that in Control, and the increases in Recovery compared with Heat. Circles denote relative interaction frequency. Bars indicate mean values of three technical replicates. e FISH results showing the association rates of $K E E 3 / K E E 4$ and of KEE7/KEE8. Bars indicate the association rates ("Methods"). Significant differences between two groups at a time were determined by the two-sided Fisher's exact test, with $p$ values adjusted by the false discovery rate method for multiple comparisons. Exact $p$ values are shown above the bars. Source data of $\mathbf{d}$ and $\mathbf{e}$ are provided as a Source Data file.

associated with TE bodies and flanking regions. We created twodimensional local rescaled pileup of chromatin interaction frequency relative to local background on heat-activated TEs or flanking regions in Control and Heat. We found that chromatin interactions in upstream and downstream flanking regions of heat-activated TEs were reduced, while chromatin interactions over TE bodies remained unaltered or became stronger (Fig. 6e). Heat-induced TE activation correlated with these chromatin organization changes (Fig. 6e, f). The results from both replicates reached the same conclusion (Fig. 6e).

In summary, chromatin interactions around TE bodies were weakened by heat stress. Heat-induced TE activation correlated with chromatin organization changes (Fig. 6e, f).

\section{Discussion}

In this study, we profiled the changes of gene and TE expression levels in response to heat and then during recovery from heat. We found that many heterochromatic TEs were activated and most of the TE activation events were transient. By performing $\mathrm{Hi}-\mathrm{C}, 3 \mathrm{C}$, and FISH, we demonstrated that heat-induced chromatin organization rearrangement with decreased long-range chromatin interactions but increased local chromatin interactions. We established that TEs were activated where chromatin organization was rearranged.

Since heat-induced TE activation is accompanied by heterochromatin decondensation, we aimed to find the correlation between heat-induced heterochromatin decondensation and heatinduced TE activation at a genome-wide scale. Our Hi-C results revealed that heat could induce genome-wide increases of shortrange chromatin interactions but decrease long-range chromatin interactions, and the interactions mediated by two prominent chromatin structures, the KNOT and the knob, were also decreased under heat stress. Importantly, we found that decreases of chromatin interactions within pericentromeric regions, the KNOT, the knob, and between knob and pericentromeric regions, and within the upstream and downstream flanking regions of heat-activated TEs correlated well with TE activation by heat. In mouse, the release of the murine endogenous retroviral element (MuERV-L/MERVL) family of TEs drives the establishment of insulating TAD boundaries during early embryogenesis ${ }^{37}$, suggesting that heterochromatin decondensation could be a consequence of TE activation. However, the upregulation of TEs other than MuERV-L/MERVL are not coupled with changes in chromatin organization ${ }^{37}$. Furthermore, the activation of TEs in the Arabidopsis mom1 mutant are not associated with changes in chromatin organization ${ }^{5}$. Thus, chromatin organization rearrangement does not always follow TE activation and it is possible that heterochromatin decondensation gives rise to TE activation.
In an interspecific hybrid of $A$. thaliana and its closest relative A. lyrata, the interactions among KEEs became weaker ${ }^{28}$. Here we found another scenario in which the interactions among KEEs are reduced. We found that the interactions within the knob and between the knob and the left pericentromere of chromosome 4 were reduced. We also found that triangle structures, which overlap with small chromatin loops, are prevalent in the Arabidopsis. So far, the mechanisms underlying the formation of the KNOT, the knob, small chromatin loops, and triangle structures are unclear. In mammals, distinct TADs and loops are formed by cohesin through loop extrusion until CCCTC-binding factor (CTCF) is encountered ${ }^{38}$. Arabidopsis REC8 (a core component of cohesin) is enriched in both centromeres and heterochromatin during meiosis ${ }^{39}$. When REC8 is depleted, many transposons are activated $^{39}$. While CTCF has no direct homolog in plants, rice $\mathrm{Hi}-\mathrm{C}$ analysis revealed that the plant-specific proteins TCP and bZIP recognize motifs embedded in TADs and contribute to the formation of TADs ${ }^{3,40}$. It will be interesting to determine whether REC8, TCP, and bZIP are involved in the formation of the KNOT and the knob.

We found that heat stress induces an increase in nuclear size (Supplementary Fig. 4c). However, heat not only induces weakening of some chromosome interactions, but also induces strengthening of some other chromatin interactions and HIT4 is required for heat-induced TE activation ${ }^{16,17}$, suggesting that heatinduced chromatin reorganization and TE activation are active processes. They are not merely a result of bigger nuclei.

Histone $\mathrm{H} 1$ binds to linker DNA wrapped away from the nucleosome core particle to form the chromatosome ${ }^{41}$. The establishment of higher-order chromatin structures requires histone $\mathrm{H} 1^{41}$. Disassociation of $\mathrm{H} 1.2$ from chromatin leads to global chromatin decondensation during mouse cell reprogramming to pluripotency ${ }^{42}$. Histone $\mathrm{H} 1$ can regulate transposon activity in Drosophila ovaries ${ }^{43}$ and in cancer cells ${ }^{44}$. In Arabidopsis, histone $\mathrm{H} 1$ is required for chromatin condensation, but the function of histone $\mathrm{H} 1$ in TE silencing is still unclear ${ }^{24-26}$. Here, we found that heat-activated TEs showed enrichment of H1.1 and H1.2 under normal conditions (Supplementary Fig. 2d). Interestingly, H1.1 and H1.2 were downregulated by heat, as revealed by our RNA-Seq and RT-qPCR data (Supplementary Fig. 1c, e). We speculate that the downregulation of histone $\mathrm{H} 1$ may contribute to heat-induced chromatin reorganization and TE activation.

In summary, we examined heat-induced TE activation and $3 \mathrm{D}$ genome reorganization at high resolution and found a strong correlation between TE activation and chromatin reorganization. Further studies are required to determine whether a cause-andeffect relationship can be established between these two events and dissect the molecular mechanisms upstream and downstream of these two events. 
a

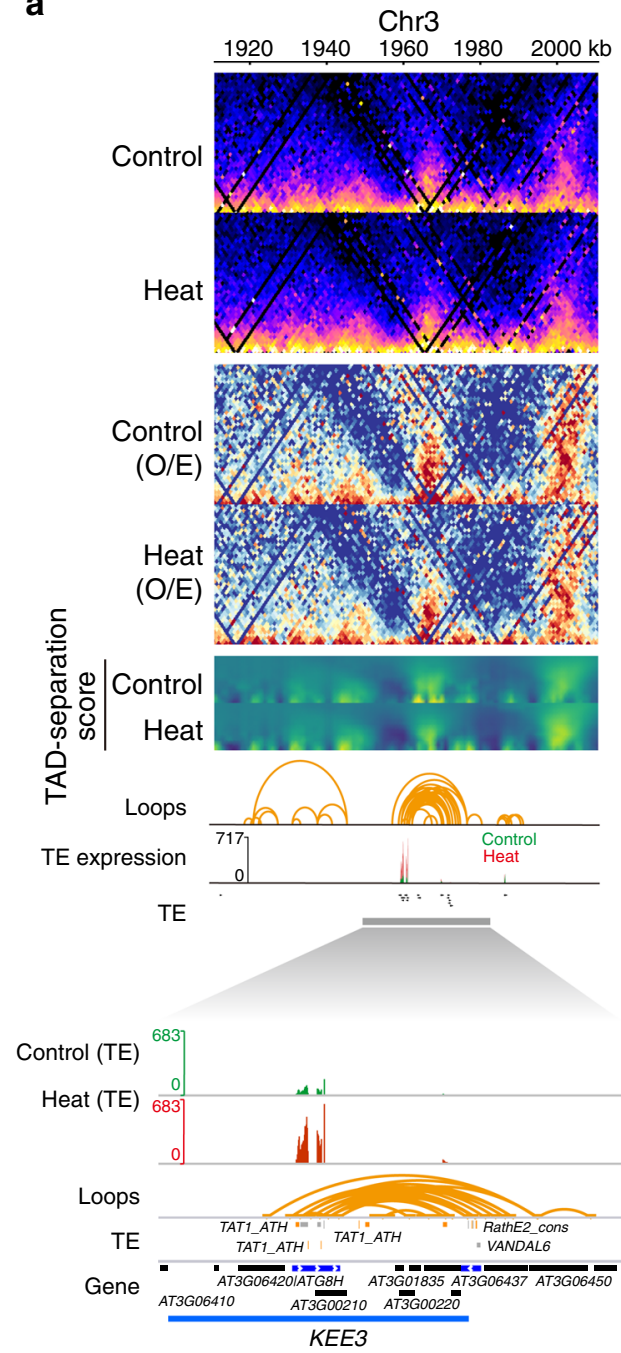

b $\begin{array}{cccc}\text { Chr3 } \\ 3080 & 3100 & 3120 \quad 3140 \quad 3160 \mathrm{~kb}\end{array}$
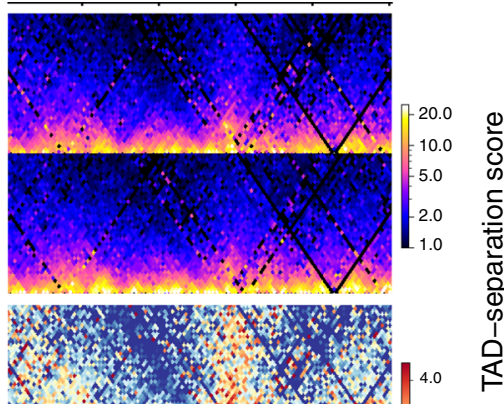
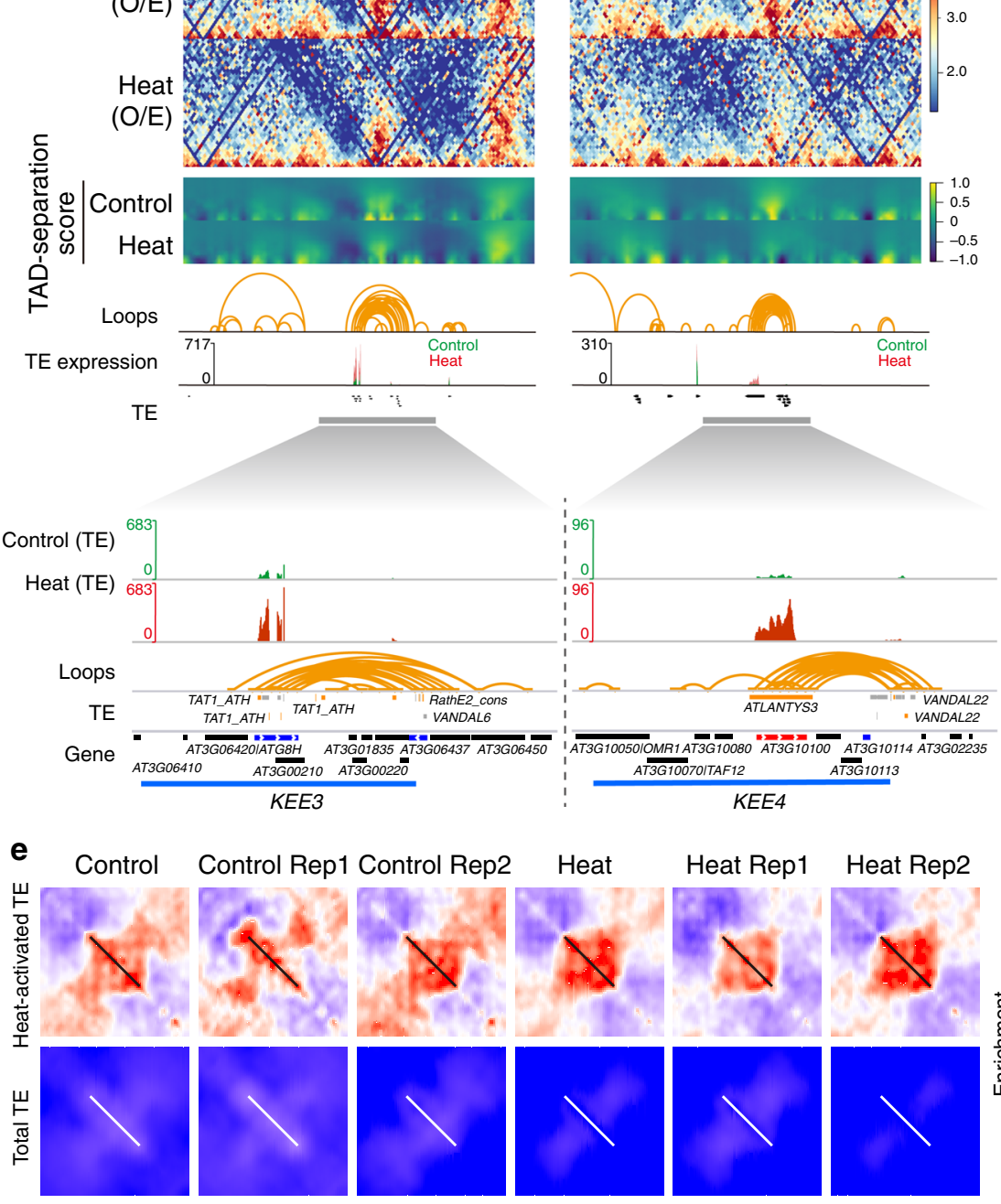
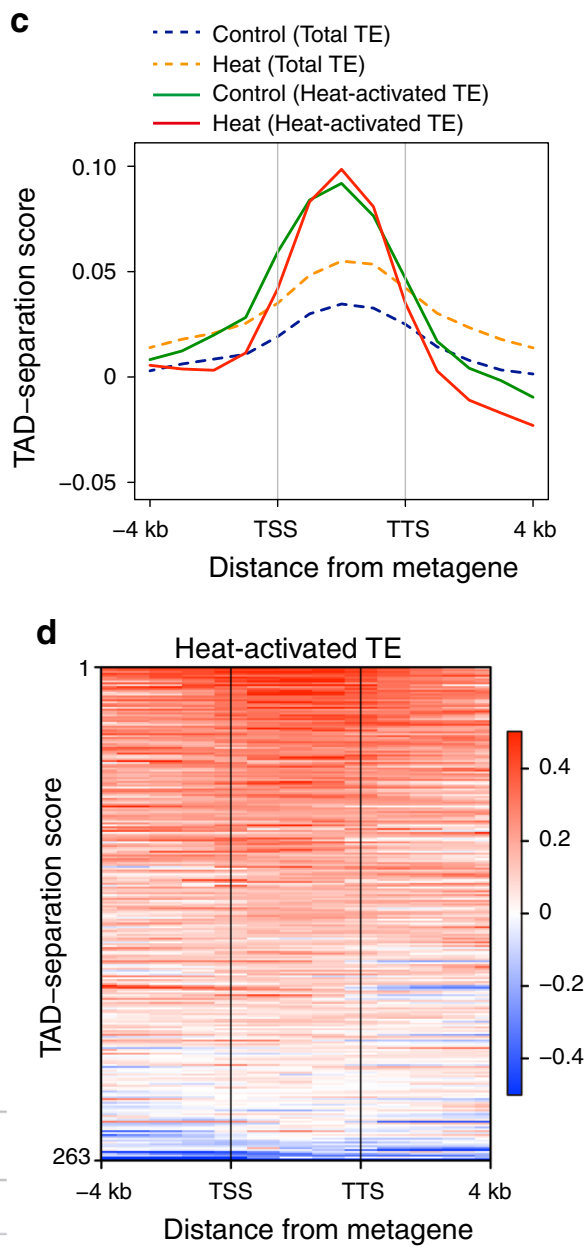

$\mathbf{f}$

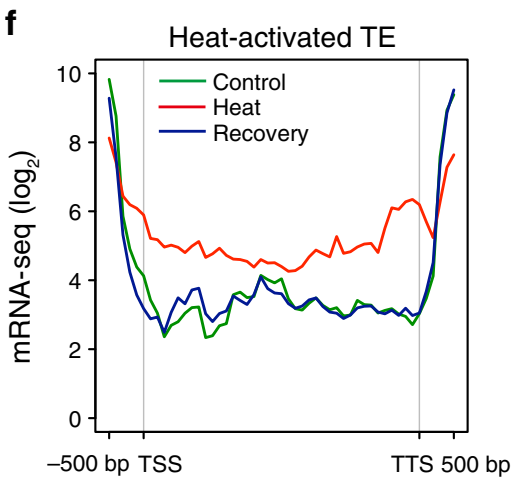

Distance from metagene

Fig. 6 Chromatin organization rearrangement revealed by high-resolution Hi-C correlates with heat-induced TE expression. a, b Changes of local chromatin organization and TE expression at KEE3 and KEE4 loci after heat treatment. Two 100 kb regions (depth: 50 kb) containing KEE3 and KEE4, respectively, are shown. The top panels are heatmaps showing natural logarithm of $1 \mathrm{~kb}$ Knight and Ruiz (KR) normalized contact frequency in Control and Heat. Below are heatmaps (O/E) showing natural logarithm of observed/expected contact frequency in Control and Heat at $1 \mathrm{~kb}$ resolution. Below are heatmaps showing TAD-separation scores at different window sizes for each $\mathrm{Hi}-\mathrm{C}$ genomic bin in Control and Heat. Loops were collected and transformed from a previous study ("Methods"). TE expression track shows the expression levels of TEs in Control and Heat. Their expression levels in Control and Heat are under the same scale and overlayered together. Only TE expression is shown. The expression of PCGs is not shown ("Methods"). The expanded panels show TE expression and the corresponding chromatin loops over KEE3 and KEE4. c Metagene plot of TAD-separation scores over bodies of heatactivated TEs and total TEs and flanking regions. Only TEs with length over 1,000 bp are considered. TSS transcription start site, TTS transcription termination site. $\mathbf{d}$ Heatmap of TAD-separation scores over bodies of 263 heat-activated TEs and flanking regions in Control. e Local rescaled pileup of chromatin organization over bodies of heat-activated TEs (upper panel) and total TEs (lower panel) in Control, Heat, and different biological replicates. The values are normalized by a local background ("Methods"). The diagonal bars are the locations of scaled heat-activated TEs (upper panel) and total TEs (lower panel). f Metagene plot of RNA-Seq read coverage over bodies of heat-activated TEs in Control, Heat, and Recovery. Heat stress can activate heterochromatin-associated transposon elements (TEs). Here, the authors show that heat stress leads to global rearrangement of 3D genome and TEs activation closely correlates with 3D chromatin organization rearrangement in Arabidopsis. 


\section{Methods}

Plant material and growth conditions. Wide-type Col-0 was used for all of the experiments. Plants were grown on 1/2 Murashige and Skoog plates with $1 \%$ sucrose and $0.7 \%$ agar. For the heat treatment, 7 -day-old seedlings were placed in an incubator set at alternating temperatures of 37 and $22^{\circ} \mathrm{C}, 12 \mathrm{~h}$ each, for 3 days with a $12 \mathrm{~h}$ photoperiod. For recovery, seedlings that underwent heat treatment were grown under normal growth conditions (a constant $22^{\circ} \mathrm{C}$ with a $12 \mathrm{~h}$ photoperiod) for an additional 3 days (Supplementary Fig. 1a).

Analysis of TE expression by RT-qPCR. We extracted total RNA from seedlings using TRIzol reagent (Invitrogen) and removed DNA contamination by using DNase (Ambion). Pure RNA $(2 \mu \mathrm{g})$ was used for first-strand cDNA synthesis with the PrimeScript II first-strand cDNA synthesis kit (Takara). The resultant cDNA reaction mixture was then diluted five times, and $1 \mu \mathrm{L}$ was used as the template in a $25 \mu \mathrm{L}$ PCR reaction with iQ SYBR Green Supermix. ACTIN7 was used as an internal control and our primers are listed in Supplementary Data 6.

Immunofluorescence. We performed immunofluorescence as previously described ${ }^{45}$. Briefly, seedlings ( $2 \mathrm{~g}$ ) were cut into pieces and homogenized in the cold $\left(4{ }^{\circ} \mathrm{C}\right)$ with $2 \mathrm{~mL}$ nucleic extraction buffer $(10 \mathrm{mM}$ Tris- $\mathrm{HCl}(\mathrm{pH} 9.5), 10 \mathrm{mM} \mathrm{KCl}$, $250 \mathrm{mM}$ sucrose, $3.97 \mathrm{mM}$ spermidine, $9.86 \mathrm{mM}$ spermine, $0.1 \% \beta$-mercaptoethanol, and $1 \%$ Triton X-100). To fix the nuclei, $2 \mathrm{~mL}$ of $8 \%$ formaldehyde was added to the homogenate and the sample was incubated at $4{ }^{\circ} \mathrm{C}$ for $1 \mathrm{~h}$ with constant shaking. The sample was filtered sequentially through 100,53 , and $20 \mu \mathrm{m}$ filters and then centrifuged at $4{ }^{\circ} \mathrm{C}$. The pellet was resuspended in $300 \mu \mathrm{L}$ of nucleic extraction buffer with $4.27 \%$ sucrose and then added on top of $300 \mu \mathrm{L}$ of nucleic extraction buffer with $29 \%$ sucrose. After centrifugation at $4{ }^{\circ} \mathrm{C}$ for $30 \mathrm{~min}$, the white pellet was resuspended in $40 \mu \mathrm{L}$ of nucleic extraction buffer. Nuclei were placed on histological slides and then immunostained with anti-H3K27me1 (07-448; Millipore; 1:200 dilution) and then incubated with rabbit Alexa488(A23220; Abbkine; 1:200 dilution) conjugated secondary antibodies for $2 \mathrm{~h}$ at $37^{\circ} \mathrm{C}$. After PBS washes, DNA was counterstained using DAPI in Prolong Gold (Invitrogen). Nuclei were observed with a Nikon A1RSi+ confocal microscope. For each sample, about 300 nuclei were analyzed.

Chromatin conformation capture. Chromatin conformation capture (3C) assays were performed according to previously described procedures ${ }^{46}$. Briefly, seedlings (1-3 g) were cross-linked with $1 \%$ formaldehyde in NIB buffer [ $20 \mathrm{mM}$ HEPES, $\mathrm{pH}$ 8.0, $250 \mathrm{mM}$ sucrose, $1 \mathrm{mM} \mathrm{MgCl}, 5 \mathrm{mM} \mathrm{KCl}, 40 \%$ (v/v) glycerol, $0.25 \%$ (v/v) Triton X-100, $0.1 \mathrm{mM}$ phenylmethanesulfonyl fluoride (PMSF), $0.1 \mathrm{mM} \mathrm{DTT}]$ and $2 \mathrm{M}$ glycine was used to quench formaldehyde. Seedlings were then washed with $\mathrm{dd}_{2} \mathrm{O}$. After removing water and being dried in air, different seedlings in equal weight were ground into fine powder in liquid nitrogen. Nuclei were isolated and then treated with SDS. Chromatin was digested by HindIII and DNA ends of digested chromatin were then ligated by T4 DNA ligase. DNA was purified after reverse cross-linking. Then $3 \mathrm{C}$ quantitative PCR was performed. The sequences of primers were as previously reported ${ }^{5}$ and listed in Supplementary Data 6. A couple of primers with opposite directions which could amplify a length of $100 \mathrm{bp}$ sequence across one of the HindIII restrict site of $S D C$ were used as a normalization control.

Fluorescence in situ hybridization (FISH). We performed FISH as previously described ${ }^{47}$. Briefly, four bacterial artificial chromosome (BAC) clones were used as probes to monitor chromosome pairing rates in Control, Heat, and Recovery, respectively. F24P17, T22K18, F10M6, and F21C20 represent KEE3, KEE4, KEE7, and KEE8, respectively. The BAC DNA was labeled with either biotin or digoxigenin and biotin-labeled probes were detected with Alexa Fluor 488 streptavidin while digoxigenin-labeled probes were detected with rhodamine anti-digoxigenin. We counterstained nuclei with DAPI and acquired chromosome images with a Zeiss A2 fluorescence microscope with a micro-charge-coupled device camera. Paring rates of two BAC clones were defined as 2 (pairing), 1 (half-pairing), and 0 (no pairing). The association rate was calculated as the sum of all the pairing events divided by twofold of the total nuclei number. Distance between two closest probes was measured by Zeiss ZEN lite.

For a post-hoc analysis, we analyzed KEE association data using pairwise Fisher's exact tests with $p$ values adjusted by the false discovery rate method for multiple comparisons ${ }^{48}$.

RNA-Seq and data analysis. We extracted total RNA from seedlings by using the RNeasy Plant Mini Kit (Qiagen) with RNase-Free DNase (Qiagen). Poly-A RNASeq library preparation and high-throughput sequencing were performed by Beijing Novogene Co. Ltd and we used the NEBNext ${ }^{\circledR}$ Ultra $^{\text {at }}$ RNA Library Prep Kit for Illumina ${ }^{\oplus}$ (NEB, USA) to generate sequencing libraries, following the manufacturer's recommendations. The libraries were sequenced by the Illumina Hisec 4000 platform, generating paired-end, $150 \mathrm{bp}$ reads.

Adapter sequences and poor-quality reads were removed by trim_galore (https://www.bioinformatics.babraham.ac.uk/projects/trim_galore/) with flags - paired and -length 70 (minimum read length after trimming). Then the clean reads were mapped to the Arabidopsis reference genome (TAIR10, https://www.arabidopsis.org) guided by Araport11 annotations ${ }^{49}$ (https://www. araport.org) and using TopHat $2^{50}$ with default parameters, except that options -min-intron-length and -max-intron-length were set to 20 and 5000 respectively. Reads were then sorted, indexed and compressed by SAMtools ${ }^{51}$ and only uniquely mapped reads were kept for the following analysis by SAMtools ${ }^{51}$. Here multi-mapped reads were disregarded because it is difficult to determine their real locations, whereas uniquely mapped reads provide sufficient coverage and mappability. We generated Bigwig files by using bam2wig.py with options $-\mathrm{u}$ (skip non-unique hit reads) and - wigsum $=10,000,000,000$ from $\mathrm{RseQC}^{52}$.

TEs with more than $20 \%$ of their regions overlapping with genes were removed because of the difficulty of their reads assignment. First, we used featureCounts ${ }^{53}$, with the option $-p$ (fragments will be counted rather than reads), to count over meta-features, like genes or TEs, and then used DESeq $2^{54}$ for differential expression analysis of genes and TEs. The expression levels of genes and TEs in different groups (Heat vs. Control and Recovery vs. Heat) were compared with strict criteria $(q<0.05$ and fold change $>2)$ and reproducibility was revealed with PCA, using the expression levels of the top 1,000 DEGs. Expression heatmaps were generated by using ComplexHeatmap ${ }^{55}$ in R. Also, in $\mathrm{R}$, we used gtrellis ${ }^{58}$ to illustrate the chromosome-wide distribution of released TEs. The TE family and superfamily information was downloaded from TAIR10 (https://www.arabidopsis.org) and screenshots of typical loci were generated using the Integrative Genomics Viewer (IGV) ${ }^{56}$. GO analyses were conducted by agriGO V2.0 (http://systemsbiology.cau.edu.cn/agriGOv2/index.php) with default parameters.

Hi-C library construction and sequencing. Annoroad Gene Technology Co., Ltd (Beijing, China) constructed and sequenced our Hi-C libraries as follows. Briefly, the aerial parts of 10-day-old seedlings were collected, cut into pieces, and crosslinked in $2 \%$ formaldehyde solution for $15 \mathrm{~min}$ and then glycine was added to quench the cross-linking reaction. After the nuclei were isolated, the chromatin was digested ${ }^{57}$. To isolate the interacting chromatin fragments, the cross-linked DNA fragments were end-labeled with biotin-14-dCTP and end joined ${ }^{58}$. Proteins were removed with proteinase $\mathrm{K}$ and the DNA was purified using phenol and chloroform. Biotin-14-dCTP was removed from non-ligated fragments with T4 DNA polymerase, then the ligated fragments were sonicated into fragments of 200-600 bp each and the DNA fragments with biotin labeling were enriched using streptavidin $\mathrm{C} 1$ magnetic beads. A-tails were added by Klenow (exo-) and Illumina paired-end sequencing adapters were ligated to DNA fragments using a ligation mix. After 12-14 cycles of PCR, the Hi-C libraries were sequenced by an Illumina HiSeq X Ten instrument with $2 \times 150$ bp reads.

Hi-C sequencing data processing by HiC-Pro. Fastp ${ }^{59}$ was used to filter lowquality reads and trim adapters from raw Hi-C sequencing reads, then $\mathrm{Hi}-\mathrm{C}$ sequencing data mapping, reads pairs filtering, and identification of valid ligations were performed in $\mathrm{HiC}-\mathrm{Pro}^{60}$. To begin, each clean mate from the read pairs was independently mapped to the Arabidopsis reference genome, TAIR10 (https:// www.arabidopsis.org), by bowtie ${ }^{61}$ using an end-to-end method to avoid linear constraints between read pairs. A two-step approach in HiC-Pro was used to rescue a small number of chimeric reads which had at least one read spanning over the junction of the ligation product. Singletons, low-quality mapped reads, and multiple mapped reads were discarded, and only unique mapped reads were kept for downstream analysis. Based on the sequence of reference genome and the restriction enzyme, each mapped read was assigned to a restriction fragment. By excluding both dangling-end and self-circle pairs, invalid ligation products were separated from valid pairs. We used the valid read pairs that comprised two different restriction fragments to construct our downstream Hi-C contact map. After removing read pairs from PCR duplicates, we calculated Hi-C resolution based on previous definition ${ }^{32}$ and then constructed $\mathrm{Hi}-\mathrm{C}$ contact maps with equal-sized bins for different resolutions. HiC-Pro contact matrixes and bins were transformed into different formats for specific analysis. Hi-C contact matrixes at different resolutions were imported into HiCExplorer ${ }^{62}$ (https://github.com/deeptools/ HiCExplorer/) in a format h5 by hicTransform with fixed bins. Cooler files were generated by hicTransform.

Hi-C map normalization and comparisons. We normalized Hi-C contact maps in HiCdat ${ }^{33}$, correcting our Hi-C data biases with the implicit bias-removal method: iterative coverage normalization ${ }^{63}$. To assess differences between $\mathrm{Hi}-\mathrm{C}$ replicates, in-house generated $\mathrm{Hi}-\mathrm{C}$ datasets, and previously published $\mathrm{Hi}-\mathrm{C}$ datasets, multiple functions in $\mathrm{HiCDat}^{33}$ were used, including f.HiC.correlation.matrix, and f.principle.component.analysis.and.features. To compare two Hi-C heatmaps, we calculated relative differences as the difference of matched bins between the two samples, further divided by the mean contact interaction frequencies between the two samples ${ }^{29}$. Comparisons between Control, Control Rep1, Control Rep2, Heat, Heat Rep1, and Heat Rep2 were performed.

To quantitatively detect the difference in chromatin organization between samples, distance-dependent decay of interaction frequency was calculated by a modified version of hicPlotDistVsCounts from HiCExplorer ${ }^{62}$. The decay plots were visualized by ggplot2 (http://ggplot2.tidyverse.org). The IDE was defined 
as the slope of a linear fit to the decreased mean interaction frequency with increased genomic distance and it was used to quantify the differences of chromatin organizations among different genomic compartments or different samples ${ }^{4}$. To further examine the effects of heat on chromatin organization, linear regression on averaged raw decay curves was performed to calculate IDEs. To compare changes in KEE-mediated chromatin interactions at $1 \mathrm{~kb}$ resolution, we used HOMER ${ }^{30}$ to calculate interaction scores (interactions per hundred square kilobases per billion mapped reads) among pairwise interactions of KEEs.

To define and compare chromatin compartments in Control and Heat, PCA analyses of Hi-C were performed by HOMER (http://homer.ucsd.edu/homer/ interactions2/index.html). To begin, The PCA calculation was performed on individual chromosome arms separated by centromeres. A balanced and distance normalized matrix was created using window size of $60 \mathrm{~kb}$ binned every $20 \mathrm{~kb}$ for each individual chromosome arm, reporting observed/expected matrix. Then, a correlation matrix was generated by calculating correlation coefficient. The matrix was further analyzed by the prcomp function from $\mathrm{R}$, and the eigenvalues for each $20 \mathrm{~kb}$ region along the $\mathrm{PC} 1$ were assigned to each region (PC1 values). To make PC1 values more comparable across chromosome arms, the PC1 values of each chromosome arm were also scaled by their standard deviation. For each chromosome, the orientation of PC1 values is determined by H3K4me3 ChIP-Seq peaks to ensure positive $\mathrm{PC} 1$ values associated with $\mathrm{A}$ compartments and negative PC1 values with B compartments. Differences in PC1 values $(\triangle \mathrm{PC} 1)$ in Heat relative to Control conditions were calculated by subtractBedGraphs.pl in HOMER with option - center. Chromatin compartmentation saddle plot and chromatin strength were calculated by GENOVA (https://github.com/robinweide/GENOVA/) using $20 \mathrm{~kb}$ contact matrix.

Comparison between $\mathrm{Hi}-\mathrm{C}$ data and TE expression data. All Hi-C datasets were trimmed to the same sequencing depth by hicNormalize from HiCExplorer ${ }^{62}$. The raw matrix was further transformed into normalized $\mathrm{Hi}-\mathrm{C}$ contact matrix by $\mathrm{KR}$ balancing method by hicCorrectMatrix from HiCExplorer ${ }^{62}$. Hi-C observed/ expected matrixes were then generated by hicTransform using a method of obs_exp_lieberman. Figure 6a, b and Supplementary Fig. 14 were generated by pyGenomeTracks (https://github.com/deeptools/pyGenomeTracks/) based on HiC data, RNA-Seq data, and annotations. Configure files were submitted into github (https://github.com/Linhua-Sun/Ath_Heat_Hi-C). Enlarged expression tracks were generated by IGV. TE expression tracks were extracted by bedops ${ }^{64}$

TAD-separation score is used to define the degree of segregation between left and right part of each Hi-C genomic bin. In our study, we found that local triangles are widespread and prominent in Arabidopsis Hi-C map, indicating the prevalence of local 3D chromosome structures in Arabidopsis. To test whether heat-activated TEs are associated with local chromosome structures, we applied hicFindTADs from HiCExplorer to calculate TAD-separation scores with options - minDepth 3000 - maxDepth 30000 - step 1000 - thresholdComparisons 0.05 --delta 0.01 --correctForMultipleTesting fdr at $1 \mathrm{~kb}$ resolution in Control and Heat. Metagene plot and associated heatmap of TAD-separation scores were generated by seqplots (http://seqplots.ga/). And then local rescaled pileup plots normalized by local backgrounds were generated by coolpup.py ${ }^{65}$ (https://github.com/Phlya/ coolpuppy/) with options - minshift 10000 -maxshift 50000 -rescale -local rescale_size 87.

Public genomic data analysis. Various Arabidopsis ChIP-seq and DNase-seq datasets were downloaded from the Plant Chromatin State Database ${ }^{66}$ and the average occupied score for each element was calculated by bedmap ${ }^{64}$ with option -wmean. ChIP-seq occupied scores among different groups were then evaluated by pairwise Mann-Whitney $U$-tests with Bonferroni-Holm method for multiple comparisons ${ }^{67}$. Used ChIP-Seq, MNase-Seq, and ChAP-Seq datasets were summarized in Supplementary Data 7.

Chromatin loops were collected and transformed from previously published study ${ }^{31}$. The significant chromatin loops were called from regions except near centromeres (Chr1:13,698,787-15,897,560; Chr2:2,450,002-5,500,000;

Chr3:11,298,762-14,289,014; Chr4:1,800,001-5,150,000; Chr5:10,999,995-13,332,770). To compare these small chromatin loops and heat-activated TEs, we used bedtools pairToBed with option - type ospan.

Reporting summary. Further information on research design is available in the Nature Research Reporting Summary linked to this article.

\section{Data availability}

Data supporting the findings of this work are available within the paper and its Supplementary Information file. A reporting summary for this Article is available as a Supplementary Information file. The datasets generated and analyzed during the current study are available from the corresponding author upon request. Raw $\mathrm{Hi}-\mathrm{C}$ sequencing reads that support the findings of this study have been submitted to the NCBI Sequence Read Archive under accession number PRJNA545383. RNA-Seq sequencing reads have been submitted to Gene Expression Omnibus under accession number GSE132415. The source data underlying Figs. 2b, c, 4a, d, 5d, e, and Supplementary Figs. 1d, 3a, b, 4b, c, $9 \mathrm{~d}$, and $12 \mathrm{~b}$ are provided as a Source Data file.

\section{Code availability}

All related scripts have been uploaded into github.

Received: 14 August 2019; Accepted: 30 March 2020;

Published online: 20 April 2020

\section{References}

1. Lieberman-Aiden, E. et al. Comprehensive mapping of long-range interactions reveals folding principles of the human genome. Science 326, 289-293 (2009).

2. Dekker, J., Rippe, K., Dekker, M. \& Kleckner, N. Capturing chromosome conformation. Science 295, 1306-1311 (2002).

3. Doğan, E. S. \& Liu, C. Three-dimensional chromatin packing and positioning of plant genomes. Nat. Plants 4, 521-529 (2018).

4. Grob, S., Schmid, M. W. \& Grossniklaus, U. Hi-C analysis in Arabidopsis identifies the KNOT, a structure with similarities to the flamenco locus of Drosophila. Mol. Cell 55, 678-693 (2014).

5. Feng, S. et al. Genome-wide Hi-C analyses in wild-type and mutants reveal high-resolution chromatin interactions in Arabidopsis. Mol. Cell 55, 694-707 (2014).

6. Brennecke, J. et al. Discrete small RNA-generating loci as master regulators of transposon activity in Drosophila. Cell 128, 1089-1103 (2007).

7. Grob, S. \& Grossniklaus, U. Invasive DNA elements modify the nuclear architecture of their insertion site by KNOT-linked silencing in Arabidopsis thaliana. Genome Biol. 20, 120 (2019).

8. Mcclintock, B. Chromosome morphology in Zea Mays. Science 69, 629-629 (1929).

9. Fransz, P. F. et al. Integrated cytogenetic map of chromosome arm 4s of $A$. thaliana: structural organization of heterochromatic knob and centromere region. Cell 100, 367-376 (2000).

10. CSHL/WUGSC/PEB, Arabidopsis Sequencing Consortium. The complete sequence of a heterochromatic island from a higher eukaryote. Cell 100, 377-386 (2000).

11. Grob, S., Schmid, M. W., Luedtke, N. W., Wicker, T. \& Grossniklaus, U. Characterization of chromosomal architecture in Arabidopsis by chromosome conformation capture. Genome Biol. 14, R129 (2013).

12. Lang-Mladek, C. et al. Transgenerational inheritance and resetting of stressinduced loss of epigenetic gene silencing in Arabidopsis. Mol. Plant 3, 594-602 (2010).

13. Pecinka, A. et al. Epigenetic regulation of repetitive elements is attenuated by prolonged heat stress in Arabidopsis. Plant Cell 22, 3118-3129 (2010).

14. Tittel-Elmer, M. et al. Stress-induced activation of heterochromatic transcription. Plos Genet. 6, e1001175 (2010).

15. Bourguet, P. et al. A role for MED14 and UVH6 in heterochromatin transcription upon destabilization of silencing. Life Sci. Alliance 1, e201800197 (2018).

16. Wang, L.-C., Wu, J.-R., Hsu, Y.-J. \& Wu, S.-J. Arabidopsis HIT4, a regulator involved in heat-triggered reorganization of chromatin and release of transcriptional gene silencing, relocates from chromocenters to the nucleolus in response to heat stress. N. Phytol. 205, 544-554 (2015).

17. Wang, L.-C. et al. Arabidopsis HIT4 encodes a novel chromocentre-localized protein involved in the heat reactivation of transcriptionally silent loci and is essential for heat tolerance in plants. J. Exp. Bot. 64, 1689-1701 (2013).

18. Cavrak, V. V. et al. How a retrotransposon exploits the plant's heat stress response for its activation. Plos Genet. 10, e1004115 (2014).

19. Henderson, I. R. \& Jacobsen, S. E. Tandem repeats upstream of the Arabidopsis endogene SDC recruit non-CG DNA methylation and initiate siRNA spreading. Genes Dev. 22, 1597-1606 (2008).

20. Silveira, A. B. et al. Extensive natural epigenetic variation at a de novo originated gene. Plos Genet. 9, e1003437 (2013).

21. Ito, H. et al. An siRNA pathway prevents transgenerational retrotransposition in plants subjected to stress. Nature 472, 115-119 (2011).

22. Pietzenuk, B. et al. Recurrent evolution of heat-responsiveness in Brassicaceae COPIA elements. Genome Biol. 17, 209 (2016)

23. Yokthongwattana, C. et al. MOM1 and Pol-IV/V interactions regulate the intensity and specificity of transcriptional gene silencing. EMBO J. 29, 340-351 (2010).

24. Rutowicz, K. et al. Linker histones are fine-scale chromatin architects modulating developmental decisions in Arabidopsis. Genome Biol. 20, 157 (2019).

25. He, S., Vickers, M., Zhang, J. \& Feng, X. Natural depletion of histone H1 in sex cells causes DNA demethylation, heterochromatin decondensation and transposon activation. eLife 8, e42530 (2019).

26. Choi, J., Lyons, D. B., Kim, M. Y., Moore, J. D. \& Zilberman, D. DNA methylation and histone $\mathrm{H} 1$ jointly repress transposable elements and aberrant intragenic transcripts. Mol. Cell 77, 310-323.e7 (2020). 
27. Lajoie, B. R., Dekker, J. \& Kaplan, N. The hitchhiker's guide to Hi-C analysis: practical guidelines. Methods 72, 65-75 (2015).

28. Zhu, W. et al. Altered chromatin compaction and histone methylation drive non-additive gene expression in an interspecific Arabidopsis hybrid. Genome Biol. 18, 157 (2017).

29. Moissiard, G. et al. MORC family ATPases required for heterochromatin condensation and gene silencing. Science 336, 1448-1451 (2012).

30. Wang, C. et al. Genome-wide analysis of local chromatin packing in Arabidopsis thaliana. Genome Res. 25, 246-256 (2015).

31. Liu, C. et al. Genome-wide analysis of chromatin packing in Arabidopsis thaliana at single-gene resolution. Genome Res. 26, 1057-1068 (2016).

32. Rao, S. S. P. et al. A 3D map of the human genome at kilobase resolution reveals principles of chromatin looping. Cell 159, 1665-1680 (2014).

33. Schmid, M. W., Grob, S. \& Grossniklaus, U. HiCdat: a fast and easy-to-use HiC data analysis tool. BMC Bioinform. 16 (2015).

34. Heinz, S. et al. Simple combinations of lineage-determining transcription factors prime cis-regulatory elements required for macrophage and B cell identities. Mol. Cell 38, 576-589 (2010).

35. Hsieh, T.-H. S. et al. Mapping nucleosome resolution chromosome folding in yeast by Micro-C. Cell 162, 108-119 (2015).

36. Ramírez, F. et al. High-resolution TADs reveal DNA sequences underlying genome organization in flies. Nat. Commun. 9, 189 (2018).

37. Kruse, K. et al. Transposable elements drive reorganisation of 3D chromatin during early embryogenesis. Preprint at https://www.biorxiv.org/content/ 10.1101/523712v1 (2019).

38. Vian, L. et al. The energetics and physiological impact of cohesin extrusion. Cell 173, 1165-1178.e20 (2018).

39. Lambing, C. et al. Interacting genomic landscapes of REC8-cohesin, chromatin and meiotic recombination in Arabidopsis thaliana. Plant Cell, https://doi.org/10.1105/tpc.19.00866 (2020).

40. Liu, C., Cheng, Y.-J., Wang, J.-W. \& Weigel, D. Prominent topologically associated domains differentiate global chromatin packing in rice from Arabidopsis. Nat. Plants 3, 742-748 (2017).

41. Fyodorov, D. V., Zhou, B.-R., Skoultchi, A. I. \& Bai, Y. Emerging roles of linker histones in regulating chromatin structure and function. Nat. Rev. Mol. Cell Biol. 19, 192-206 (2018).

42. Christophorou, M. A. et al. Citrullination regulates pluripotency and histone H1 binding to chromatin. Nature 507, 104-108 (2014).

43. Iwasaki, Y. W. et al. Piwi modulates chromatin accessibility by regulating multiple factors including histone $\mathrm{H} 1$ to repress transposons. Mol. Cell $\mathbf{6 3}$, 408-419 (2016).

44. Izquierdo-Bouldstridge, A. et al. Histone $\mathrm{H} 1$ depletion triggers an interferon response in cancer cells via activation of heterochromatic repeats. Nucleic Acids Res 45, 11622-11642 (2017).

45. Jasencakova, Z., Meister, A., Walter, J., Turner, B. M. \& Schubert, I. Histone $\mathrm{H} 4$ acetylation of euchromatin and heterochromatin is cell cycle dependent and correlated with replication rather than with transcription. Plant Cell 12, 2087-2100 (2000).

46. Louwers, M., Splinter, E., van Driel, R., de Laat, W. \& Stam, M. Studying physical chromatin interactions in plants using Chromosome Conformation Capture (3C). Nat. Protoc. 4, 1216-1229 (2009).

47. Zhang, D. et al. Molecular cytogenetic characterization of the Antirrhinum majus genome. Genetics 169, 325-335 (2005)

48. Benjamini, Y. \& Hochberg, Y. Controlling the false discovery rate: a practical and powerful approach to multiple testing. J. R. Stat. Soc. Ser. B Methodol. 57, 289-300 (1995).

49. Cheng, C.-Y. et al. Araport11: a complete reannotation of the Arabidopsis thaliana reference genome. Plant J. 89, 789-804 (2017).

50. Kim, D. et al. TopHat2: accurate alignment of transcriptomes in the presence of insertions, deletions and gene fusions. Genome Biol. 14, R36 (2013).

51. Li, H. et al. The Sequence Alignment/Map format and SAMtools. Bioinformatics 25, 2078-2079 (2009).

52. Wang, L., Wang, S. \& Li, W. RSeQC: quality control of RNA-seq experiments. Bioinformatics 28, 2184-2185 (2012).

53. Liao, Y., Smyth, G. K. \& Shi, W. featureCounts: an efficient general purpose program for assigning sequence reads to genomic features. Bioinformatics 30, 923-930 (2014).

54. Love, M. I., Huber, W. \& Anders, S. Moderated estimation of fold change and dispersion for RNA-seq data with DESeq2. Genome Biol. 15, 550 (2014).

55. Gu, Z., Eils, R. \& Schlesner, M. Complex heatmaps reveal patterns and correlations in multidimensional genomic data. Bioinformatics 32, 2847-2849 (2016).

56. Robinson, J. T. et al. Integrative genomics viewer. Nat. Biotechnol. 29, 24-26 (2011).
57. Dong, Q. et al. Genome-wide Hi-C analysis reveals extensive hierarchical chromatin interactions in rice. Plant J. 94, 1141-1156 (2018).

58. Belton, J.-M. et al. Hi-C: a comprehensive technique to capture the conformation of genomes. Methods 58, 268-276 (2012).

59. Chen, S., Zhou, Y., Chen, Y. \& Gu, J. fastp: an ultra-fast all-in-one FASTQ preprocessor. Bioinformatics 34, i884-i890 (2018).

60. Servant, N. et al. HiC-Pro: an optimized and flexible pipeline for Hi-C data processing. Genome Biol. 16, 259 (2015).

61. Langmead, B. \& Salzberg, S. L. Fast gapped-read alignment with Bowtie 2. Nat. Methods 9, 357-359 (2012).

62. Wolff, J. et al. Galaxy HiCExplorer: a web server for reproducible Hi-C data analysis, quality control and visualization. Nucleic Acids Res. 46, W11-W16 (2018).

63. Zhang, Y. et al. Spatial organization of the mouse genome and its role in recurrent chromosomal translocations. Cell 148, 908-921 (2012).

64. Neph, S. et al. BEDOPS: high-performance genomic feature operations. Bioinformatics 28, 1919-1920 (2012).

65. Flyamer, I. M., Illingworth, R. S. \& Bickmore, W. A. Coolpup.py: versatile pileup analysis of Hi-C data. Bioinformatics, https://doi.org/10.1093/ bioinformatics/btaa073.

66. Liu, Y. et al. PCSD: a plant chromatin state database. Nucleic Acids Res. 46 , D1157-D1167 (2018).

67. Holm, S. A simple sequentially rejective multiple test procedure. Scand. J. Stat. 6, 65-70 (1979)

\section{Acknowledgements}

This work was supported by the National Key R\&D Program of China (2018YFE0204700 and 2016YFA0500800) and the National Natural Science Foundation of China (31970614) to W.Q. Most of the Hi-C bioinformatic analyses were performed on the High-Performance Computing platform at the Center for Life Sciences (Peking University).

\section{Author contributions}

W.Q. and H.H. conceived and designed the project. Y.J., X.L., and Q.L. performed the experiments and managed sequencing. Z.X. and Z.C. performed the FISH experiments. L.S. performed all bioinformatics analyses. L.S., Y.J., X.L., and Q.L. analyzed the data. L.S and X.L. wrote the paper draft. Y.J., X.L., and Q.L. contributed to paper discussion. W.Q. H.H., and D. W. revised the paper. W.Q. and H.H. supervised the work.

\section{Competing interests}

The Authors declare no Competing Interests.

\section{Additional information}

Supplementary information is available for this paper at https://doi.org/10.1038/s41467 020-15809-5.

Correspondence and requests for materials should be addressed to H.H. or W.Q.

Peer review information Nature Communications thanks Chang Liu, Daniel Zilberman and the other, anonymous, reviewer(s) for their contribution to the peer review of this work

Reprints and permission information is available at http://www.nature.com/reprints

Publisher's note Springer Nature remains neutral with regard to jurisdictional claims in published maps and institutional affiliations.

Open Access This article is licensed under a Creative Commons Attribution 4.0 International License, which permits use, sharing, adaptation, distribution and reproduction in any medium or format, as long as you give appropriate credit to the original author(s) and the source, provide a link to the Creative Commons license, and indicate if changes were made. The images or other third party material in this article are included in the article's Creative Commons license, unless indicated otherwise in a credit line to the material. If material is not included in the article's Creative Commons license and your intended use is not permitted by statutory regulation or exceeds the permitted use, you will need to obtain permission directly from the copyright holder. To view a copy of this license, visit http://creativecommons.org/ licenses/by/4.0/

(C) The Author(s) 2020 